\title{
1 Dopamine Neurons Evaluate Natural Fluctuations in Performance Quality
}

2 Alison Duffy, ${ }^{1}$ Kenneth W. Latimer, ${ }^{2}$ Jesse H. Goldberg, ${ }^{3}$ Adrienne L. Fairhall, ${ }^{1,}{ }^{*}$ and Vikram

3 Gadagkar ${ }^{3,4,5, *}$

$4 \quad{ }^{1}$ Department of Physiology and Biophysics, University of Washington, Seattle, WA 98195, USA

$5 \quad$ 2Department of Neurobiology, University of Chicago, Chicago, IL 60637, USA

$6{ }^{3}$ Department of Neurobiology and Behavior, Cornell University, Ithaca, NY 14853, USA

$7 \quad{ }^{4}$ Department of Neuroscience, Zuckerman Mind Brain Behavior Institute, Columbia University,

$8 \quad$ New York, NY 10027, USA

$9 \quad{ }^{5}$ Lead Contact

$10{ }^{*}$ Correspondence: fairhall@uw.edu (A.L.F), vikram.gadagkar@columbia.edu (V.G.)

\section{SUMMARY}

13 Many motor skills are learned by comparing ongoing behavior to internal performance

14 benchmarks. Dopamine neurons encode performance error in behavioral paradigms where

15 error is externally induced, but it remains unknown if dopamine also signals the quality of

16 natural performance fluctuations. Here we recorded dopamine neurons in singing birds and

17 examined how spontaneous dopamine spiking activity correlated with natural fluctuations in

18 ongoing song. Antidromically identified basal ganglia-projecting dopamine neurons correlated

19 with recent, and not future, song variations, consistent with a role in evaluation, not

20 production. Furthermore, dopamine spiking was suppressed following the production of

21 outlying vocal variations, consistent with a role for active song maintenance. These data show 
22 for the first time that spontaneous dopamine spiking can evaluate natural behavioral

23 fluctuations unperturbed by experimental events such as cues or rewards.

\section{KEYWORDS}

26 Dopamine, Birdsong, Gaussian Process Model, Generalized Linear Model, Performance

27 Prediction Error, Natural Behavior, Motor Skill Learning, Skill Maintenance, Basal Ganglia,

28 Ventral Tegmental Area

\section{INTRODUCTION}

31 Dopamine (DA) is associated with fluctuations in future movements as well as the outcomes of

32 past ones. During spontaneous behavior, DA activity can be phasically activated before a

33 movement (da Silva et al., 2018; Hamilos et al., 2020), or can ramp as an animal approaches

34 reward (Hamid et al., 2016; Howe et al., 2013). DA neurons can also signal a reward prediction

35 error (RPE) during reward seeking, where phasic signals represent the value of a current

36 outcome relative to previous outcomes (Schultz et al., 1997). It remains poorly understood how

37 spontaneous DA activity relates to natural fluctuations in behavior that are independent of

38 experimentally induced rewards or perturbations.

Zebra finches provide a tractable model to study the role of DA in natural behavior.

40 First, they sing with a significant amount of trial-to-trial variability, but the overall stereotypy of

41 the song allows renditions to be accurately compared. Second, they have a discrete neural

42 circuit (the song system) that includes a DA-basal ganglia (BG) loop (Figures $1 \mathrm{~A}$ and $1 \mathrm{~B}$ ) that is

43 necessary for song learning and maintenance (Brainard and Doupe, 2000; Hisey et al., 2018; 
44 Hoffmann et al., 2016; Xiao et al., 2018). Third, BG projecting DA neurons signal performance

45 prediction error (PPE) during singing: they exhibit pauses following worse-than-predicted

46 outcomes caused by distorted auditory feedback (DAF), and they exhibit phasic bursts following

47 better-than-predicted outcomes when predicted distortions do not occur (Figures 1A-1D)

48 (Gadagkar et al., 2016). Yet one limitation of this study was that song quality was controlled

49 with an external sound (DAF, Figure 1C), so it remains unclear if the DA system is simply using

50 song timing to build expectations about an external event (DAF), or if it also evaluates the

51 quality of natural fluctuations (Figure $1 \mathrm{E}$ ), which would be necessary for natural song learning.

52 Furthermore, this experimental paradigm did not test if DA activity was associated with

53 upcoming syllables, consistent with a premotor signal.

To test DA's role in natural behavior, we recorded from DA neurons in the ventral

55 tegmental area (VTA, Figure 1B), and examined how spiking activity correlated with natural

56 song fluctuations (Figure 1E). First, if DA activity following externally distorted and undistorted

57 song (Figure 1D) truly reflects a function of the DA system in performance evaluation, then DA

58 activity should correlate with recent song fluctuations (Figure 1E). Second, DAF-associated error

59 signaling was previously only observed in a small subclass of 'VTA error' neurons, most of which

60 projected to Area X, the BG nucleus of the song system. 'VTA other' neurons were defined by the

61 absence of an error signal during singing. We hypothesize that the VTA error population will carry

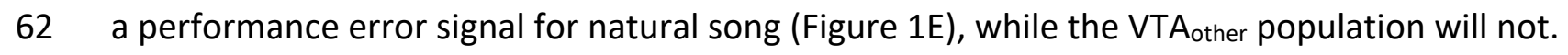

63 Thus, we ask in this analysis: do VTA neuron activity patterns relate to fluctuations in natural

64 song? If so, what is the structure of these relationships, and do they relate to a performance

65 evaluation framework, a premotor framework, or both? 
To answer these questions, we first parameterized natural song into a low dimensional

67 set of time-varying song features. We then agnostically fit the relationship between rendition-

68 to-rendition variations in song features and spike counts at local time-steps in song across a

69 range of song segment-spike window latencies and identified if and when song feature

70 variations predicted spike counts. Finally, we characterized both the timing and the form of

71 these predictive fits. We find that the activity of the VTA error, but not the VTA other, neuronal

72 population encodes fluctuations in natural song in a manner consistent with a performance

73 evaluation signal. These results show that basal ganglia-projecting DA neurons may provide

74 continuous evaluation of natural motor performance independent of external rewards or

75 perturbations.

76

77 RESULTS

79 A Gaussian Process Model Approach Reveals Song-Spike Relationships

80 We sought to identify how VTA spiking varied with natural fluctuations in song syllables. To

81 identify relationships between natural song fluctuations and VTA spiking, we chose an eight-

82 dimensional, time-varying representation of song based on established song parameterizations

83 (Figure 2A; see STAR Methods). For each neuron, we identified song syllables and binned both

84 song feature values and spike counts in sliding windows to search for relationships between

85 song fluctuations and spike counts at different latencies (Figure 2B; see STAR methods). We

86 combined all eight song features and binned spike counts into a single multi-dimensional

87 Gaussian Process (GP) regression model (two features shown for illustration) to quantify 
whether song feature fluctuations predicted spike counts (Figures 2C and S1-S3; see STAR

Methods). This strategy flexibly identifies the most relevant dimensions of song variation within a single model. Specifically, we computed an $r^{2}$ value for each model fit using leave-one-out cross validation to assess how well variations in song features predicted spike counts (Figure $2 C)$. Values of $r^{2}>0$ indicates that song feature variations across renditions can predict spike counts; the larger the $r^{2}$ value, the more predictive the song-spike relationship in the model.

94 Finally, we fit the full model to many song-spike latencies and thus built a matrix of $r^{2}$ values for

95 each neuron's response to song fluctuations, with each $r^{2}$ value in the matrix representing one

96 full model fit (one feature shown for illustration) between a song window-spike window pair

97 (Figure 2D).

\section{Process}

101 Using the GP model approach described above, we asked if significant relationships between natural song fluctuations and VTA neuron spiking exist, and if so, at what song-spike latencies they occur. If VTA spiking is predictive of upcoming syllable fluctuations in a premotor fashion,

104 then significant relationships would be observed at negative lags. Alternatively, if VTA spiking is 105 playing an evaluative function, then variations in spike counts should follow variations in syllable acoustic structure, and relationships should be observed at positive lags. Based on past

107 work (Gadagkar et al., 2016), an evaluation signal is predicted to occur at a positive lag of 50

108 ms with a duration range of 0 to 150 ms. Figure 3A shows an example VTAerror neuron's song- 
110 window (song window width $=35 \mathrm{~ms}$ ) aligned to syllable onset $(t=0)$. The $x$-axis is the latency,

111 defined as the time between the song window midpoint and the spike window midpoint (spike

112 window width $=100 \mathrm{~ms}$ ). Colored pixels in the $\mathrm{r}^{2}$ matrix indicate that song feature fluctuations

113 predict spike counts $\left(r^{2}>0\right)$; greyscale pixels indicate that song feature fluctuations do not

114 predict spike counts $\left(r^{2}<=0\right)$. The pink box indicates the song-spike latencies $(0-150 \mathrm{~ms})$ where

115 we expect to see evaluation-like relationships based on the DAF experimental results (Figure

116 1D). We assessed the significance of finding predictive fits by shuffling entire spike trains

117 relative to song renditions and refitting our model across all latencies and song windows

118 (Figures 3A bottom and S4; see STAR Methods). This population method of shuffling the data

119 preserves the underlying temporal correlation structure of song and spiking while randomizing

120 the song-spike relationship and allowed us to assess the significance of the entire set of model

121 fits and account for multiple comparisons (see STAR Methods). The bottom matrix in Figure 3A

122 shows the $r^{2}$ values from one such randomized shuffle of the same neuron's activity patterns.

123 Positive $r^{2}$ values were found to be less frequent in the shuffled data (one-sided z-test: $p<$

$1240.01)$

We then analyzed the temporal relationship between song features and spiking by

126 comparing the latency distribution for all song window-spike window pairs within the $r^{2}$ matrix

127 in which song features predicted spike counts $\left(r^{2}>0\right)$ to the latency distribution for pairs

128 without predictive relationships $\left(r^{2}<=0\right)$ (Figure 3B). The latencies of the predictive fits were

129 clustered within the expected error evaluation range (0-150 ms) (Figure 3B). Figure 3C shows

130 the result of the same analysis performed across all the VTAerror neurons in our dataset $(\mathrm{N}=19)$.

131 In Figure 3C, the blue line indicates the song-spike latency distribution for which song 
132 fluctuations predict spike counts $\left(r^{2}>0\right)$ while the black line and grey shading are the mean and

133 standard deviation of the same latency distributions across all randomized population shuffles.

134 The true data showed a large peak within the expected PPE latency range (3.74 standard

135 deviations from the mean, one-sided z-test: $p<0.01$; see STAR Methods). We found that song

136 fluctuations are most predictive of spike counts 0-100 ms after the song fluctuation occurs,

137 consistent with a PPE-like signal based on our previous DAF experiments (Figure 1D). In

138 addition, across the population of VTA $A_{\text {error }}$ neurons there were significantly more predictive fits

139 within the PPE latency window than expected by chance ( $p<=0.01$; see STAR Methods). Thus,

140 the timing and frequency of the predictive song-spike relationships was remarkably consistent

141 with a PPE-like response to natural song variations.

We next performed the identical analysis on a population of VTA other neurons $(\mathrm{N}=23)$,

143 which did not show an error-like response in previous DAF experiments (Figure 1D). The

144 number of predictive song-spike relationships from this population was also significantly larger

145 than expected by chance (bootstrap test, $p<=0.01$, see STAR Methods). However, unlike the

146 VTAerror neurons, the predictive relationships from this population did not cluster within the PPE

147 latency range, nor did the variance of the distribution significantly deviate from the randomized

148 latency distributions ( $p=0.21$; Figure 3D; see STAR Methods). Thus, consistent with results from

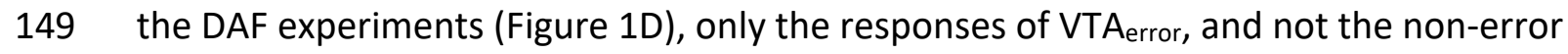

150 responsive VTA $_{\text {other, }}$ neuron population were predicted by natural song fluctuations within the

151 expected PPE latency range with significantly increased frequency. The same neurons that

152 exhibited error responses to the DAF sound exhibited significant relationships with natural 
153 syllable fluctuations. Remarkably, both the DAF-induced error and the natural fluctuations were

154 at a similar latency with respect to song.

156 The Form of the Predominant Song-Spike Relationship for VTA

\section{Song Maintenance}

158 The hypothesis that VTAerror neurons evaluate natural song fluctuations led to further

159 predictions about the forms of these song-spike relationships. If a bird is trying to maintain the

160 acoustic structure of a syllable, then typical variations should be followed by more spikes and

161 rare, outlying syllable variations should be followed by fewer spikes (Figure 4A, top left).

162 Alternatively, if the bird is trying to modify a syllable, e.g. increase its pitch, then the

163 relationship between syllable acoustic structure and spike should be directional: spike counts

164 should peak at whatever shifted variant to which the bird aspires but is not yet consistently

165 producing (Figure 4A, right panels). We did not expect PPE-like signals to have multiple maxima

166 in a disruptive fit: we assumed there is a single 'best' version of the song at each time-step

167 (Figure 4A, bottom left).

To test these possible outcomes, we characterized tuning curve shapes of song-spike

169 relationships. For this analysis, we focused our attention on the subset of GP model fits that

170 were predictive $\left(r^{2}>0\right)$. Within this subset, we further selected single-feature fits that were also

171 predictive $\left(r^{2}>0\right.$ for the 1D feature fit). We selected this subset of song-spike relationships

172 because we are interested only in the tuning curves that might actually carry information about

173 song. We re-fit all such song-spike relationships with a generalized linear model (GLM) using

174 both linear (I-GLM) and quadratic (q-GLM) features (Figure 4B; see STAR Methods). We chose 
175 these models because the parameters can be used to directly quantify aspects of the tuning

176 curve shapes. If the song-spike fit has a single peak, the spiking response is stabilizing, and the

177 quadratic coefficient of the q-GLM is negative (Figure 4A, top left). If the song-spike fit has two

178 peaks, the spiking response is disruptive, and the quadratic coefficient of the q-GLM is positive

179 (Figure 4A, bottom left). If the song-spike fit is monotonic, the spiking response is directional,

180 and the I-GLM (with only linear features) is the more appropriate model (Figure 4A, right

181 panels). Figure 4C shows examples of predictive relationships between individual song features

182 and spike counts along with all model fits (GP, q-GLM, and I-GLM). Each point on these plots

183 represents the song feature value and the spike count for a single rendition. Specific models

184 were better fits for some distributions than others. For example, in panel 4 (Figure 4C, fourth

185 panel from left), the q-GLM produced the same model fit as the I-GLM because the quadratic

186 term added no improvement to the fit, whereas in panel 3 (Figure 4C, third panel from left), the

187 quadratic term was necessary to accurately follow the spiking response and thus the q-GLM

188 resulted in a better fit than the I-GLM.

When the spiking response is either clearly stabilizing or disruptive, the quadratic

190 coefficient of the q-GLM distinguishes between these two response types making the q-GLM

191 the better model choice. We used the Akaike Information Criterion ( $\triangle A I C)$ to compare the

192 relative success of the $\mathrm{q}-$ GLM and the I-GLM (see STAR Methods). $\triangle A I C>0$ indicates that the

193 quadratic model outperforms the linear model, considering both the likelihood of the model fit

194 and the complexity of the model used. $\triangle \mathrm{AIC}=-2$ indicates the quadratic model provides no

195 benefit over the linear model. The larger the $\triangle \mathrm{AIC}$, the better the q-GLM fit relative to the I-

196 GLM. In Figure 4D, each point represents a fit to a single song feature with an $r^{2}>0$ within a 
197 multi-dimensional model fit with an $r^{2}>0$ for the population of VTA error neurons within the expected PPE latency range. We found a greater fraction (0.78) of q-GLM fits with negative quadratic coefficients, which indicates more stabilizing tuning curves than disruptive tuning

200 curves (Figure 4D). Furthermore, when the quadratic model outperformed the linear model, 201 the fraction of stabilizing fits also increased (Figure 4E). Thus, the predictive fits from the GP

202 model had significantly more stabilizing tuning curves than disruptive when their shape was

203 better characterized as quadratic rather than linear, as we expect for a PPE signal with a single

204 best outcome (2-tailed z-test: $p<0.02)$. This finding is consistent with our hypothesis that a PPE

205 signal should respond most strongly to a single best performance of song. The $\Delta \mathrm{AIC}$ measure

206 also allowed us to examine the fraction of tuning curves that are better fit by a linear versus

207 quadratic model. The VTA error population did not differ from chance in this fraction (fraction fits

208 with $\triangle \mathrm{AIC}>0=0.43 ; 2$-tailed $\mathrm{z}$-test, $\mathrm{p}=0.34)$, consistent with a PPE signal with both directional

209 and stabilizing responses depending on the current level of song error.

211 DISCUSSION

212 Value judgements in the brain are necessary to drive appropriate changes in behavior during

213 learning. Using experimentally constrained tasks with external rewards, previous studies have

214 found that DA neurons in VTA can encode a key component of value judgement: the mismatch

215 between expected and actual reward outcomes, the reward prediction error (Schultz et al.,

216 1997). However, extending these findings to natural behavior and intrinsic reward has been a

217 challenge. Here, we made use of a novel opportunity to use an experimental context to

218 partition songbird VTA neurons into error and non-error classes and analyze their spiking 
219 responses in the context of a natural behavior (Gadagkar et al., 2016). We compared natural

220 song fluctuations at a local, within-syllable scale to variations in spike counts of VTA neurons.

221 We developed a Gaussian Process regression analysis to quantify the non-stationary spiking

222 response to variations in performance at different points in song and with different temporal

223 relationships to song. We found evidence that VTA DA neurons' activity patterns correlate with

224 variations in natural song in a manner consistent with performance evaluation: both the timing

225 and tuning properties of the DA response was consistent with a PPE-like response. This finding

226 corroborates and extends complementary discoveries of RPE signals emerging from mammalian

227 DA neurons in VTA in experimentally imposed tasks. We did not find significant temporal

228 relationships between DA and song fluctuations consistent with a premotor signal as has been

229 observed in previous studies of DA (Barter et al., 2015; Engelhard et al., 2019). Our results are

230 the first direct evidence we are aware of that DA neurons in VTA respond to fluctuations in

231 natural behavior in a manner consistent with evaluation.

Two important predictions from these PPE-like signals we find in DA VTA neurons is

233 that, one, future song renditions will shift towards song variations which correlate with the

234 peak response in the DA neurons and that, two, this shift will be accompanied by a decrease in

235 the PPE peak response. We could not address these predictions here because we analyzed

236 single recording sessions of limited duration. This will be an important direction for future work

237 and will help disambiguate the role of the VTA DA responses from other possible relationships

238 to song. We chose a pre-defined set of song features $(\mathrm{N}=8)$, which have been shown to

239 represent biologically relevant song variations in previous studies, because we focused on

240 single sessions with limited data. Future work could apply more flexible, non-parametric 
241 dimensionality reduction methods using more song renditions to better identify VTA's

242 relationship to song features that are most modulated by the bird at different points in song

243 (Goffinet et al., 2021; Kollmorgen et al., 2020).

244 While, as a population, the non-error VTA other neuron activity did not relate to song

245 fluctuations in a manner consistent with a PPE signal, many neurons exhibited correlated

246 relationships to song (Chen et al., 2021). These findings are consistent with previous studies in

247 mammals which found that both DA and non-DA neurons in VTA contribute to an RPE

248 calculation and that elements of the RPE signal are computed, in part, locally within VTA (Cohen

249 et al., 2012; Dobi et al., 2010; Ju Tian, 2016; Wood et al., 2017). Correlations with song

250 variations in this population could represent components of the PPE calculation.

This project uses the structure of an experimentally grounded characterization of

252 individual neurons' response to song-triggered, distorted auditory feedback to analyze the

253 same neurons during natural behavior. The connection to an existing experiment (Gadagkar et

254 al., 2016) as well as to a Reinforcement Learning framework (Sutton and Barto, 1998) anchors

255 our interpretations of natural behavior in a constrained laboratory paradigm and theory. The

256 unusually high stereotypy of the natural behavior we consider, zebra finch song, allows

257 reasonable inferences to be made both in the experimental and natural context about the

258 behavior of the bird and a reasonable way to characterize and align a complex, natural

259 behavior. We found a parallel relationship, including a striking temporal correspondence,

260 between the VTA error neuron activity in experimental and natural contexts that corroborates the

261 experimental finding that VTA error neurons encode time-step specific performance prediction

262 errors in song. Our analysis of natural song addresses the critique that the DAF experimental 
263 paradigm is aversive rather than perturbative and thus qualitatively different from natural song

264 evaluation. A frequent debate in neuroscience is whether artificial behavioral paradigms serve

265 as true building blocks for understanding neural activity in complex, freely behaving contexts,

266 or whether they represent a different, overly-simplified context that will not extrapolate to

267 natural behavior. This experimentally guided study of natural behavior is a fruitful direction that

268 permits the control of experimental contexts and the complexity of natural contexts to interact

269 and build upon one another.

\section{ACKNOWLEDGMENTS}

272 This work was supported by NIH/NINDS (grant\# R01NS094667), Pew Charitable Trusts, and the

273 Klingenstein Neuroscience Foundation (J.H.G.), a Simons Collaboration on the Global Brain

274 (SCGB) grant (A.L.F and A.D.), an SCGB Postdoctoral Fellowship and NIH/NINDS Pathway to

275 Independence (grant\# K99NS102520 and R00NS102520) award (V.G.).

\section{AUTHOR CONTRIBUTIONS}

278 Conceptualization, A.D., K.W.L., J.H.G., A.L.F., and V.G.; Experimental Methodology and

279 Investigation: J.H.G and V.G. Analysis Methodology, A.D., K.W.L., A.L.F., and V.G.; Software and

280 Formal Analysis: A.D., K.W.L., and A.L.F.; Writing-Original Draft, A.D. and V.G.; Writing-Review

281 and Editing, A.D., K.W.L, J.H.G., A.L.F., and V.G.; Funding Acquisition, J.H.G., A.L.F, and V.G.;

282 Supervision, J.H.G., A.L.F, and V.G.

283 DECLARATION OF INTERESTS

284 The authors declare no competing interests. 
A

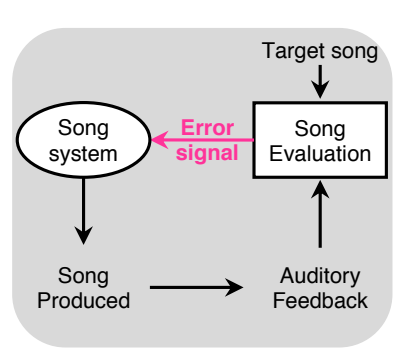

D
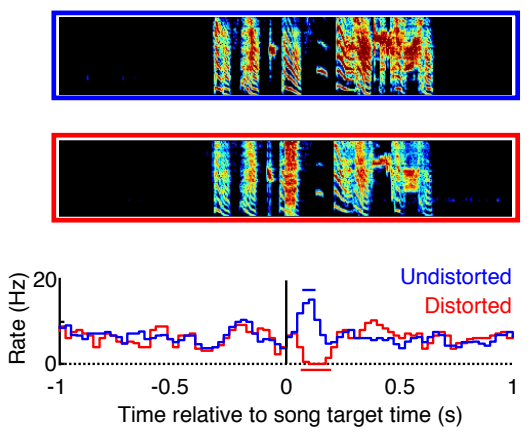

285

286

287

288

289

290

291 (labeled 'Natural') were left undisturbed.

292

(D) Left, top to bottom: example spectrograms of renditions with the target syllable undistorted

293 (enclosed in blue box) and distorted (enclosed in red box); rate histogram of distorted and

294 undistorted renditions (the horizontal bar indicates significant deviations from baseline $(p<$

295

0.05, z-test; see STAR Methods)); Right: normalized response to target syllable in VTA error $_{\text {and }}$

296
B



E Better song segment?

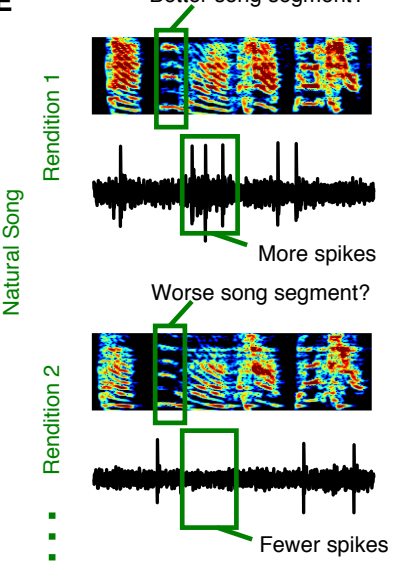

Figure 1. Experimental Identification of Performance Error in VTA DA Neurons in Singing Birds

(A) Evaluation of auditory feedback during singing is thought to produce an error signal for song learning.

(B) Basal ganglia (Area X)-projecting DA neurons from VTA were antidromically identified.

(C) Example of DAF. The target syllable was randomly distorted across motifs. All other syllables VTA other neurons (mean +- SEM, see STAR Methods). 
297 (E) The experimental results suggest a hypothesis that fluctuations in natural song should also 298 result in VTAerror responses. 
A

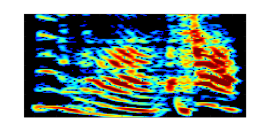

Mean frequency

Amplitude

Pitch

Entropy

FM Chromprov

AM

Aperiodicity $\mathrm{Lrn} \mathrm{N}^{\mathrm{m}}$

Goodness
of pitch

Time
B
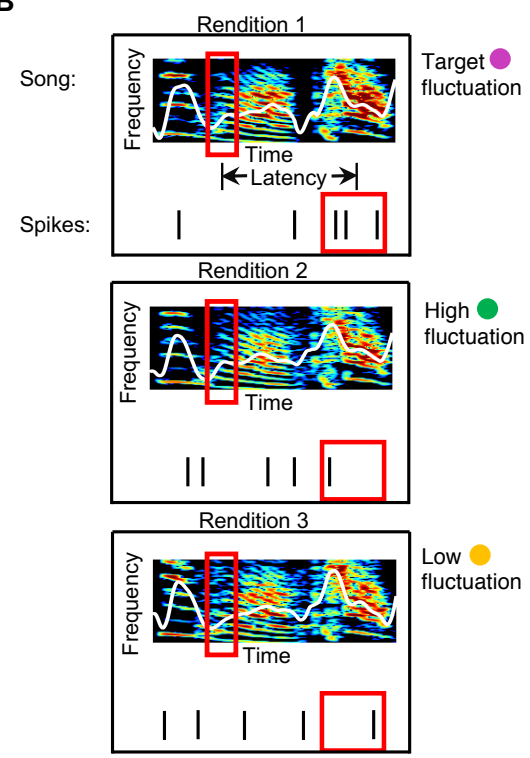

Song Feature



C

Feature combinations:
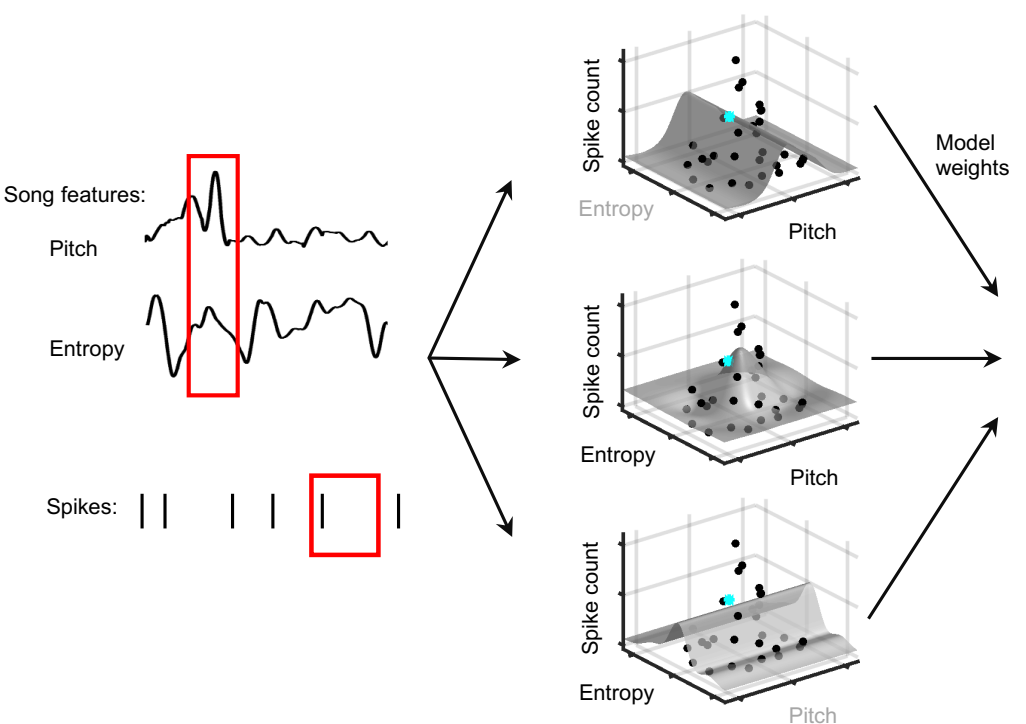

Model averaged prediction
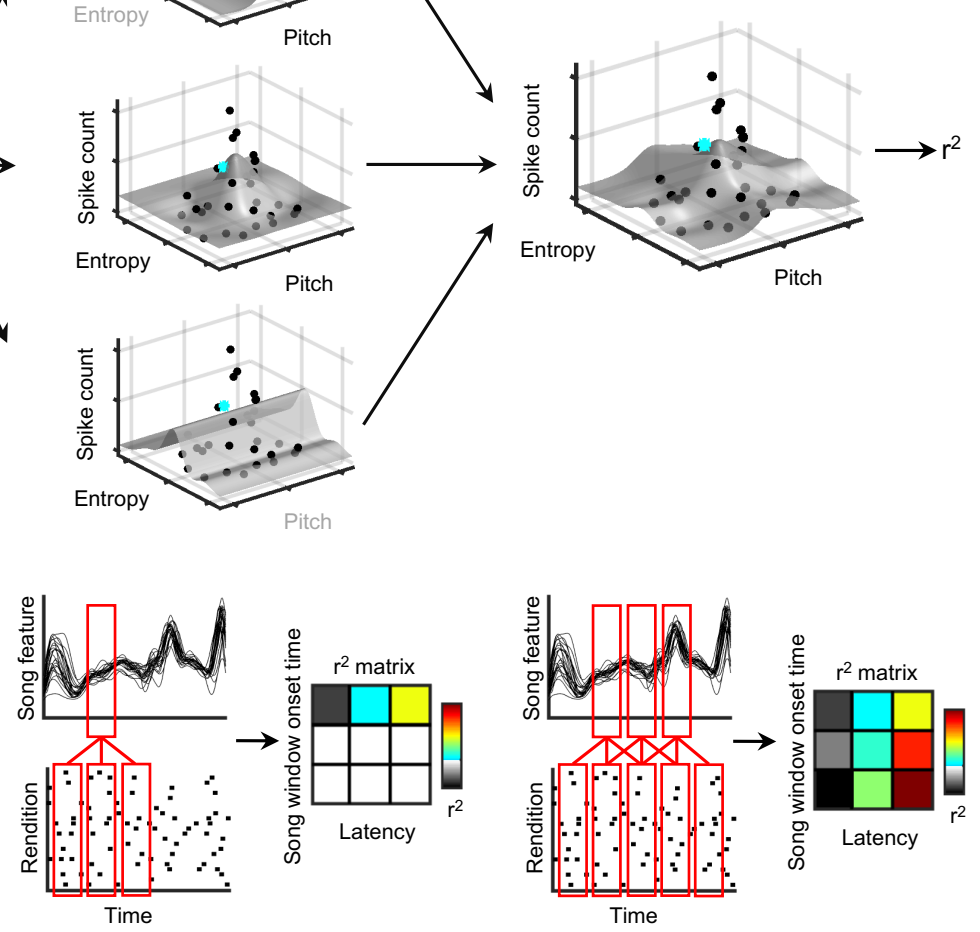


\section{Figure 2. A Gaussian Process Model Approach Reveals Song-Spike Relationships}

302 (A) Natural song was parameterized into eight time-varying song features.

303 (B) Schematic of fitting song fluctuations to spike counts within specific time windows. Local

304 feature averages (one feature shown for illustration) were used to predict local spike counts

305 using a GP model.

306 (C) Schematic of fitting a single, multivariate model using multiple song features. The

307 multidimensional model takes a weighted average of the model predictions from every

308 combination of eight song features (two shown here for illustration). The model's goodness of

309 fit was quantified by the cross-validated $r^{2}$ (see STAR Methods).

310 (D) The modeling technique shown in (B) and (C) was extended across a range of song windows

311 and song-spike latencies, thus building a matrix of $r^{2}$ values. The top panels show a sliding

312 window along the song (single feature shown for illustration). The bottom panels show the

313 time-aligned spiking activity across renditions in a raster plot. Each entry in the $r^{2}$ matrix

314 (middle panels) represents the fit between one song window and one spike window, shown

315 here connected with red lines. 
A
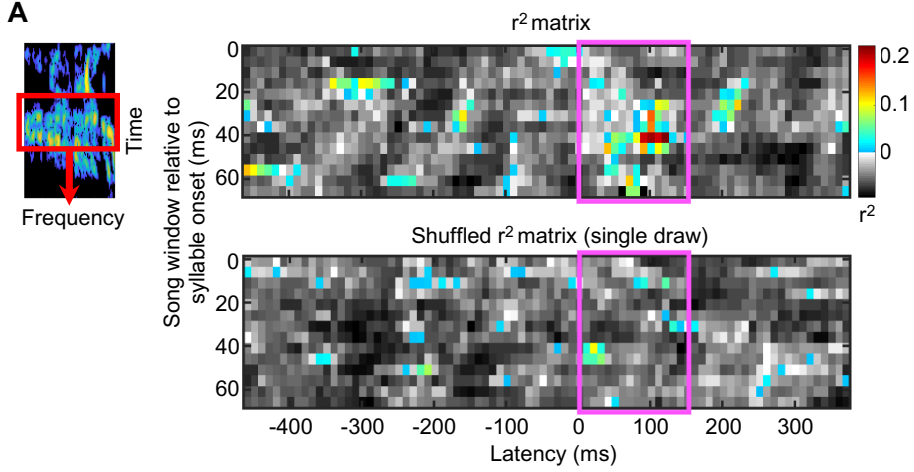

C



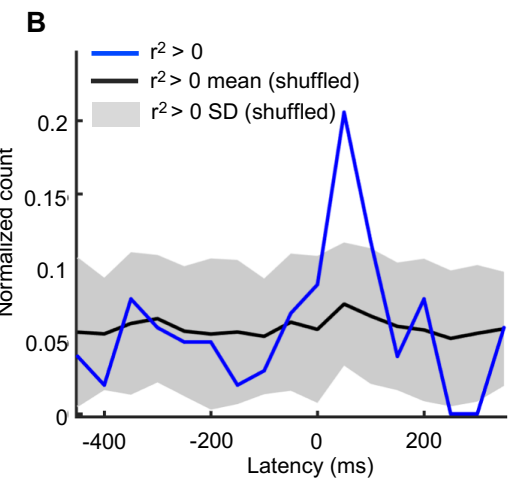

D

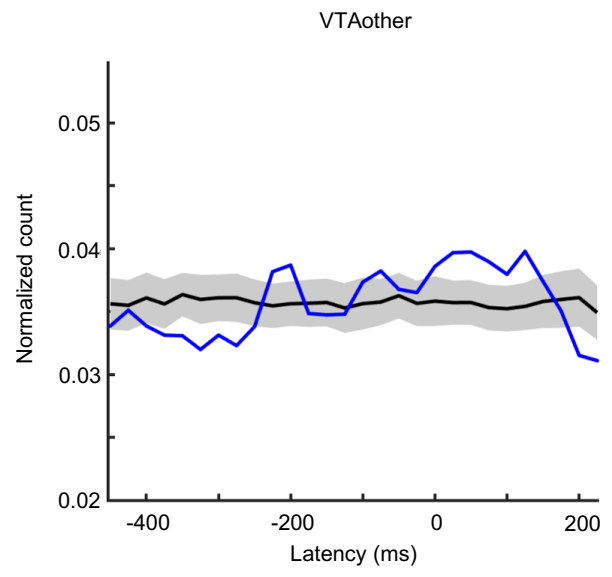

317 Figure 3. Timing of Song-Spike Relationships for VTAerror Neurons Suggests an Evaluative

Process

319 (A) Spectrogram of example syllable (top left). Heat map of $r^{2}$ values for fitted relationships

320 between local song feature averages and binned spike counts (top right). $r^{2}>0$ indicates a

321 predictive relationship. The pink box indicates the region where the latency matches the

322 hypothesized response for a PPE, 0-150 ms. The lower heat map shows an $\mathrm{r}^{2}$ matrix for a

323 shuffled version of the data (see STAR Methods).

324 (B) Histogram of latencies for predictive fits shown in (A). 
325 (C) Latency distribution of predictive fits over all VTA $A_{\text {error }}$ neurons $(\mathrm{N}=19)$ showed a significant 326 peak in the number of responses in the expected PPE time window $(* *$ indicates $p<0.01$, see 327 STAR Methods).

328 (D) Same as in (C), but for the VTA other neuron population ( $N=23)$.

329

330

331

332

333

334

335

336

337

338

339

340

341

342

343

344

345

346 
A
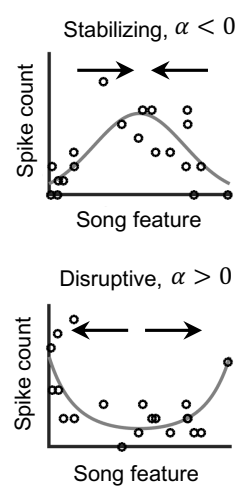

C

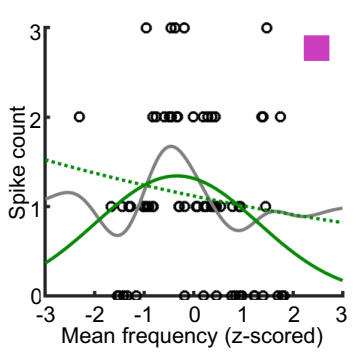

D

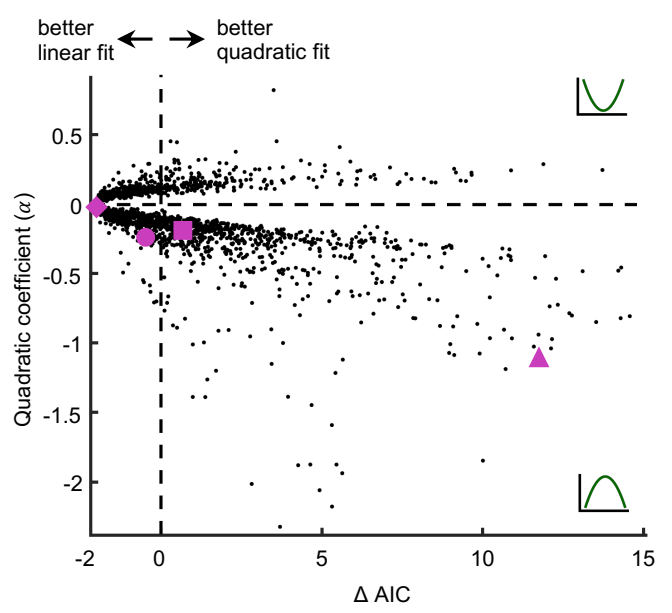

B
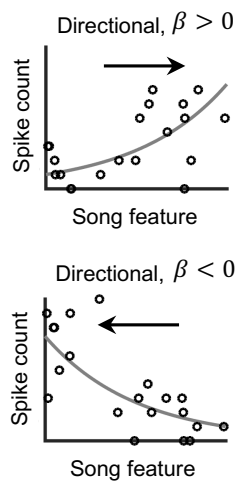

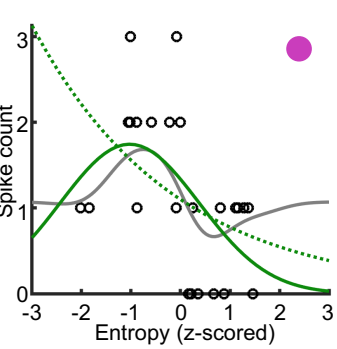

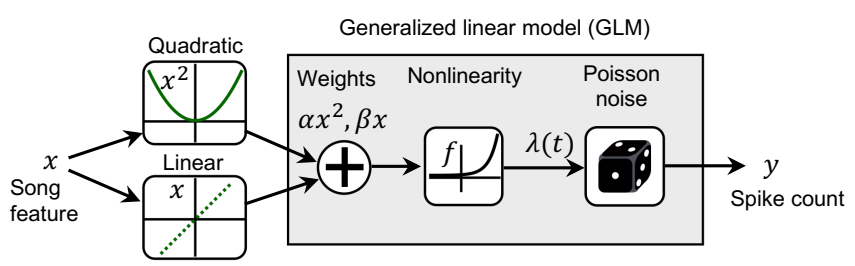



- GP model

- Quadratic GLM model

.... Linear GLM model

$\mathbf{E}$

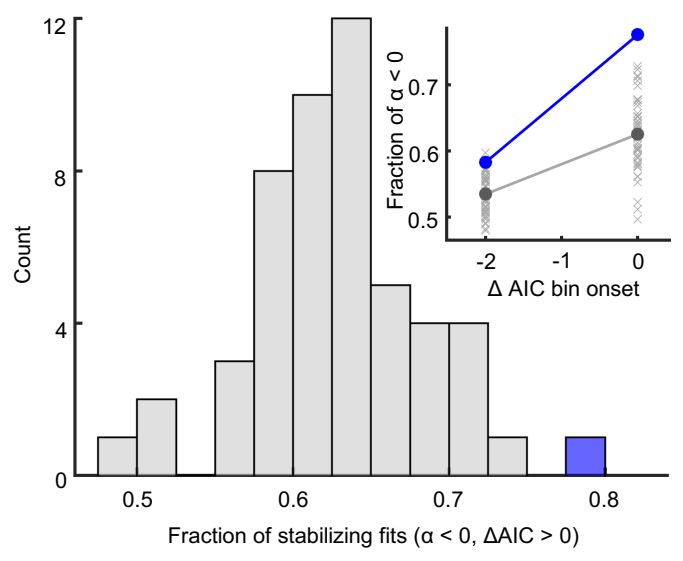

348 Figure 4. The Form of the Predominant Song-Spike Relationship for VTAerror neurons is

349 Consistent with Song Maintenance

350 (A) The form of a song-spike relationship determines how the song is being reinforced. $\alpha$ and $\beta$

351 correspond to the quadratic and linear coefficients in the GLM shown in (B).

352 (B) Schematic of the nested GLM fitting process to quantify tuning curve shape for VTAerror

353 neuron activity to natural song fluctuations. 
354 (C) Example tuning curves obtained with the GP model, I-GLM, and q-GLM between single song 355 features and spike counts for a selection of song-spike model fits. Each point on each plot 356 represents a single rendition. Pink shapes denote fit locations marked in (D).

357 (D) The quadratic coefficient for all q-GLM model fits to predictive song-spike relationships 358 (defined within the GP model) as a function of $\triangle A I C$ values in the GLM model comparison within 359 the PPE latency range. Pink shapes denote fits shown in (C).

360 (E) Fraction of stabilizing fits (negative quadratic coefficient) for all fits better described as 361 quadratic than linear $(\triangle \mathrm{AIC}>0)$ compared to shuffled population fractions. The blue point is the 362 data and each value in the gray histogram is a single fraction from an independent population 363 shuffle (see STAR Methods). The data showed a greater fraction of stabilizing fits than expected 364 by chance $(p$-value $<0.01)$. Inset: same distribution but now shown for both the binned $\Delta$ AIC $>$ 3650 group and $\triangle A I C \in[-2,0]$. The blue point is the true fraction and gray points are fractions 366 from shuffled populations (see STAR Methods). 


\section{STAR METHODS}

RESOURCE AVAILABILITY

\section{Lead Contact}

381 Further information and requests for resources should be directed to and will be fulfilled by

382 Vikram Gadagkar (vikram.gadagkar@columbia.edu).

\section{Materials Availability}

385 This study did not generate new unique reagents.

\section{Data and Code Availability}

388 The data and code generated during this study are available at (to be created code repository).

\section{EXPERIMENTAL MODEL AND SUBJECT DETAILS}

391 Subjects were 26 adult male zebra finches 74-300 days old singing undirected song. All

392 experiments were carried out in accordance with NIH guidelines and were approved by the

393 Cornell Institutional Animal Care and Use Committee. During implant surgeries, each bird was

394 anesthetized with isoflurane and a bipolar stimulation electrode was implanted into Area $\mathrm{X}$ at

395 established coordinates $(+5.6 \mathrm{~A},+1.5 \mathrm{~L}$ relative to lambda and 2.65 ventral relative to pial

396 surface; head angle 20 degrees). Intraoperatively in each bird, antidromic methods were used

397 to identify the precise part of VTA containing VTAx neurons. Next, custom made, plastic printed 
398 microdrives carrying an accelerometer, linear actuator, and homemade electrode arrays (5

399 electrodes, 3-5 MOhms, microprobes.com) were implanted into this region.

401 METHOD DETAILS

403 Syllable-targeted distorted auditory feedback

404 Detailed description of all aspects of the distorted auditory feedback (DAF) experiments is 405 described elsewhere (Gadagkar et al., 2016). Descriptions of experimental details relevant to 406 this study are presented here. Postoperative birds were placed in a sound isolation chamber 407 equipped with a microphone and two speakers which provided DAF. To implement targeted 408 DAF, the microphone signal was analyzed every $2.5 \mathrm{~ms}$ using custom Labview software. Specific 409 syllables were targeted either by detecting a unique spectral feature in the previous syllable 410 (using Butterworth band-pass filters) or by detecting a unique inter-onset interval (onset time 411 of previous syllable to onset time of target syllable) using the sound amplitude as previously

412 described (Ali et al., 2013; Hamaguchi et al., 2014; Tumer and Brainard, 2007). In both cases a 413 delay ranging from 10-200 ms was applied between the detected song segment and the target 414 time.

415 To ensure that DAF would not be perceived as an aversive stimulus, the DAF sound had the 416 same amplitude and spectral content as normal zebra finch song. For broadband DAF $(n=16$

417 birds), DAF was a broadband sound band passed at $1.5-8 \mathrm{kHz}$, the same spectral range of zebra 418 finch song (Andalman and Fee, 2009). For displaced syllable DAF ( $n=10$ birds), DAF was a 419 segment of one of the bird's own syllables displaced in time. For both types of DAF, the 
420 amplitude was carefully measured with a decibel meter (CEM DT-85A) and maintained at less

421 than $90 \mathrm{~dB}$, the average peak loudness of zebra finch song (Mandelblat-Cerf et al., 2014). This

422 ensured that DAF was not a particularly loud sound for the bird; the distorted part of the song

423 was significantly softer than the loudest parts of the song.

424 Target time in the song was defined as the median time of DAF onset across target

425 syllables; jitter of the target time was defined in each bird as the standard deviation of the

426 distribution of DAF onset times relative to the target syllable onset. Syllable truncations

427 following DAF were rare and were excluded from analysis.

428

\section{Electrophysiology}

430 Neural signals were band pass filtered $(0.25-15 \mathrm{kHz})$ in homemade analog circuits and acquired 431 at $40 \mathrm{kHz}$ using custom Matlab software. Single units were identified as Area X-projecting

432 (VTAx) by antidromic identification (stimulation intensities 50-400 $\mu \mathrm{A}, 200 \mu \mathrm{s}$ on the bipolar

433 stimulation electrode in Area X). All neurons identified as VTAx were further validated by

434 antidromic collision testing. Spike widths were computed as the trough-to-peak interval in the 435 mean spike waveform.

437 Spike sorting and analyzing responses to distorted auditory feedback

438 Spike sorting was performed offline using custom Matlab software. Firing rate histograms were 439 constructed with 25 ms bins and smoothed with a 3-bin moving average. To calculate the 440 significance of error responses (Figure $1 D$ ), spiking activity within \pm 1 second relative to target 441 onset was binned in a moving window of $30 \mathrm{~ms}$ with a step size of $2 \mathrm{~ms}$. Each bin after the 
442 target time was tested against all the bins in the previous 1 second (the prior) using a z-test

443 (Mandelblat-Cerf et al., 2014). Response onset (latency) was defined as the first bin for which

444 the next 3 consecutive bins $(6 \mathrm{~ms})$ were significantly different from the prior activity $(\mathrm{z}$-test, $\mathrm{p}<$

445 0.05); response offset was defined as the first bin after response onset for which the next 7

446 consecutive bins (14 ms) did not differ from the prior ( $p>0.05$, z-test); the response onset and

447 offset were required to bracket the maximum (undistorted) or minimum (distorted) response

448 after target time.

449

450 Parameterizing song

451 We used open source MATLAB software, Sound Analysis Pro 2011 (SAP 2011), to assemble the

452 spectrogram as well as to define and extract song features. SAP 2011 is a customized software

453 package written to analyze animal communication and is originally and most frequently used to

454 study bird song (Tchernichovski et al., 2000). We used an existing SAP feature set for our

455 parameterization because these features have been used in many previous studies to link zebra

456 finch song variations to neural activity or neuromodulator concentrations (Kao et al., 2005;

457 Leblois et al., 2010; Woolley and Kao, 2014), to study variation in song over development

458 (Deregnaucourt et al., 2004; Lipkind and Tchernichovski, 2011; Ravbar et al., 2012) and to drive

459 adult learning in DAF paradigms (Andalman and Fee, 2009; Sober and Brainard, 2009; Tumer

460 and Brainard, 2007). Therefore, we can use this form of dimensionality reduction of song

461 knowing in advance that these dimensions are behaviorally relevant to song variation in other

462 contexts. The features extracted were Wiener entropy, pitch, goodness of pitch, amplitude,

463 amplitude modulation (AM), frequency modulation (FM), mean frequency, and aperiodicity. 
464 These features result in an eight-dimensional representation of song at each time-step. We

465 further applied a moving-average filter (35 ms) to smooth the feature signals in time and

466 sampled the smoothed value every 5 ms across song.

467

468 Aligning syllables across renditions

469 To compare song across renditions, syllables were classified using custom Matlab code

470 (Gadagkar et al., 2016). Clusters of unique syllables were labelled alphabetically as 'a', 'b', 'c'

471 etc. depending on order within a rendition. The number of syllables each bird sings varies bird-

472 to-bird from 3-7 syllables. We identified syllable onsets and offsets across renditions for every

473 syllable set in which there were greater than 15 renditions of that syllable using an amplitude

474 threshold chosen to match the amplitude variance of that syllable. All alignments were further

475 checked by eye. Renditions in which alignment was ambiguous by eye were excluded from 476 analysis.

477 All syllable types (i.e. 'a' or ' $b$ ' etc.) were isolated and aligned across renditions by

478 syllable onset times. Individual syllable types have a stereotyped, characteristic duration;

479 however, there is some variation of this duration from rendition-to-rendition. In order to make

480 sure that minor differences in syllable lengths were not misaligning local syllable features at the

481 later parts of the syllable, we linearly time-warped the feature wave forms of each syllable

482 rendition such that they all lasted the median duration of that syllable type (Kao et al., 2008).

483

484

485 


\section{Parameterizing and aligning spiking activity}

487 Spike sorting was performed offline using custom MATLAB software (Gadagkar et al., 2016). For 488 every syllable, we considered the spike train $\pm 500 \mathrm{~ms}$ around the syllable onset. In order to 489 align spiking activity to the song features, we first applied the same linear time-warping map to 490 the spike train that we used to align syllables for each rendition (Kao et al., 2008). In all cases, 491 we applied this map to the time window in which the syllable took place. When possible, we 492 generated a piece-wise linear time warping map based on syllable boundaries in surrounding 493 syllables. In the time windows where there was no song with which to build a time warping 494 map, we left the spike train un-warped.

495 We binned spike counts within a sliding window (100 ms) across the 1000 ms length of 496 spike train we considered for each syllable. We chose this spike count window based on the 497 firing rate of the VTA-error neurons we considered (mean firing rate $=13 \pm 5 \mathrm{~Hz}$ ).

499 Fitting spikes to song with a Gaussian process regression

500 The goal of our analysis is to quantify non-stationary spiking responses to a time-varying 501 sensory signal, with the following characteristics. First, if VTA neuron activity encodes

502 prediction error responses to song fluctuations, these responses would be specific to the time 503 in song; an identical vocalization occurring at the beginning of the song might elicit a very 504 different response than at the middle. Second, the relevant dimensions of the signal space 505 could vary throughout the song; thus, different parameterizations of the song might provide 506 better low-dimensional representations of error-relevant song variation at different song time507 steps. Third, the form of a PPE-like tuning curve could also vary across the song. 
Towards this goal, we used a regression approach to determine if spike counts are

509 related to the variation in song. The relationship between spike counts and song is likely non-

510 linear and related to a variable number of features depending on the point in song. To address

511 this, we used a non-parametric Gaussian process (GP) regression to fit the relationship between

512 our eight song features and spike counts within single time windows (Williams, 2006).

513 There are multiple sources of model uncertainty in this task: it is unclear which and how

514 many features to use at a given point in song. Furthermore, the prediction of the model

515 depends heavily on which features are used. To address this uncertainty, we used a Bayesian

516 model averaging approach to determine the predicted spike count wherein we integrated over

517 all possible feature combinations and weighted their predictions according to their posterior

518 probability given the observed spike counts (Hoeting, 1998).

519 For each neuron, in every non-target (distorted or undistorted) syllable for which there

520 were $N \geq 15$ renditions, we sampled the smoothed song features every 5 ms across the

521 syllable and sampled spike counts in 100 ms windows every 10 ms across $1 \mathrm{~s}$ of spike train

522 centered around syllable onset. We fit the multi-dimensional GP model across all song

523 segment-spike bin pairs and generated song-spike relationships at many time latencies. We

524 additionally fit a GP model using each feature individually. For all of these fits we computed the $525 \quad r^{2}$ value.

\section{Construction of the Gaussian process regression model}

528 We modeled the relationship between the set of $\mathrm{N} \mathrm{z}$-scored song features on a single rendition

$529 \mathrm{i}, \boldsymbol{x}_{i}$, and the spike counts on that given rendition, $y_{i}$, in single time windows (e.g. the song 
530 feature values $20 \mathrm{~ms}$ after syllable onset and the spike count in a $100 \mathrm{~ms}$ window, $75 \mathrm{~ms}$ after

531 syllable onset). To address this, we used a non-parametric Gaussian process (GP) regression to

532 fit the relationship between the eight song features and spike counts across song and spike

533 window pairs (Williams, 2006). We used a Bayesian model averaging approach to combine a

534 weighted average of GP regressions using all subsets of song features into a single model

535 prediction.

536 We selected a subset of features, $M$, for a single GP regression, where feature is indexed

537 by $i=1,2, \ldots, N$ such that $\mathcal{M} \subseteq\{1,2, \ldots N\}, \mathcal{M} \neq 0, \mathrm{~N}=8$. The GP regression model for a single

538 set of $\mathcal{M}$ is:

539

540

$$
y_{i} \mid f_{\mathcal{M}} \sim \mathcal{N}\left(f_{\mathcal{M}}\left(\boldsymbol{x}_{i, \mathcal{M}}\right), \sigma^{2}\right)
$$

541

542

$$
f_{\mathcal{M}}(\cdot) \sim \mathcal{G P}\left(\mu, \omega^{2} \kappa^{(\mathcal{M})}\left(\boldsymbol{x}, \boldsymbol{x}^{\prime}\right)\right)
$$

543

544 where $\mathrm{f}$ is a function relating song features to spike rate and $\kappa$ is the covariance function and

545 defines how spike counts will be correlated with one another in feature space. We used the

546 commonly selected kernel function for $\kappa$,

547

548

$$
\kappa^{(\mathcal{M})}\left(\boldsymbol{x}, \boldsymbol{x}^{\prime}\right)=\exp \left(-\frac{\left\|\boldsymbol{x}_{\mathcal{M}}-\boldsymbol{x}_{\mathcal{M}}^{\prime}\right\|^{2}}{2 l^{2}}\right)
$$


$550 \omega^{2}$ is the GP variance term and specifies how strongly the spike counts vary as a function of the

551 song features. $\sigma^{2}$ is the variance term that captures noise in the spike count (i.e. how much the

552 spike counts vary at a single point in song feature space). The length scale, $l$, determines how

553 close points must be in feature space to have correlated spike counts. To reduce computational

554 complexity, we set $l=0.5$, for all model fits and z-scored the individual song features at each

555 time step we considered.

556 Although we do not observe f directly, the GP framework allows us to compute the

557 marginal likelihood of the data with respect to the model parameters. The likelihood of all $T$

558 renditions of spike count-song segment pairs is:

559

560

$$
p\left(y_{1: T} \mid x_{1: T}, \mathcal{M}, l, \sigma^{2}, \omega^{2}, \mu\right)=\mathcal{N}\left(\left[y_{1}, y_{2}, \ldots, y_{T}\right]^{\mathrm{T}} ;[\mu, \mu, \ldots, \mu]^{\mathrm{T}}, \omega^{2} \mathrm{~K}^{(\mathcal{M})}+\sigma^{2} I_{T}\right)
$$

561

562

$$
\mathrm{K}^{(\mathcal{M})}=\left[\begin{array}{ccc}
\kappa^{(\mathcal{M})}\left(\boldsymbol{x}_{1}, \boldsymbol{x}_{1}\right) & \cdots & \kappa^{(\mathcal{M})}\left(\boldsymbol{x}_{1}, \boldsymbol{x}_{T}\right) \\
\vdots & \ddots & \vdots \\
\kappa^{(\mathcal{M})}\left(\boldsymbol{x}_{T}, \boldsymbol{x}_{1}\right) & \cdots & \kappa^{(\mathcal{M})}\left(\boldsymbol{x}_{T}, \boldsymbol{x}_{T}\right)
\end{array}\right]
$$

563

564 where $I_{T}$ is the identity matrix of dimension $T$.

565

566

The prediction mean-squared error for the GP model is:

567

568

$$
M S E_{l o o}^{(G P)}=\frac{1}{T} \sum_{i=1}^{T}\left(y_{i}-\hat{y}_{i}\right)^{2}
$$




$$
\hat{y}_{i}=\mathbb{E}\left[y_{i} \mid \boldsymbol{x}_{/ i}, \boldsymbol{y}_{/ i}, \boldsymbol{x}_{i}\right]
$$

571

572 where $\hat{y}_{i}$ is the predicted spike count from the model for rendition $i$, and $\boldsymbol{x}_{/ i}$ and $\boldsymbol{y}_{/ i}$ are the

573 song features and spike counts for all renditions except for the $i^{\text {th }}$ rendition.

$574 \quad$ We determined the predicted spike count by applying a Bayesian model averaging

575 approach. We integrated over all possible values of $\mathcal{M}$ and then weighted their predictions

576 based on their posterior probability given observed spike counts (Hoeting, 1998):

577

578

$$
\mathbb{E}\left[y_{i} \mid \boldsymbol{x}_{/ i}, \boldsymbol{y}_{/ i}, \boldsymbol{x}_{i}\right]=\sum_{\mathcal{M} \subseteq\{1,2, \ldots N\}, \mathcal{M} \neq 0} \int_{0}^{\infty} \mathbb{E}\left[y_{i} \mid \mathcal{M}, r, \boldsymbol{x}_{/ i}, \boldsymbol{y}_{/ i}, \boldsymbol{x}_{i}\right] p\left(\mathcal{M}, r \mid \boldsymbol{x}_{/ i}, \boldsymbol{y}_{/ i}\right) d r
$$

580 where $r=\frac{\sigma^{2}}{\omega^{2}}$ is the ratio of the GP variance to the observation noise. We integrated over all 581 possible values of $\mathcal{M}$ and weighted their predictions according to their posterior probability 582 given the observed spike counts.

$$
\left.\mathbb{E}\left[y_{i} \mid \mu, \mathcal{M}, r, \boldsymbol{x}_{/ i}, \boldsymbol{y}_{/ i}, \boldsymbol{x}_{i}\right]=\mathrm{K}_{/ i, i}^{(\mathcal{M})}\right)^{\mathrm{T}}\left(\mathrm{K}_{/ i, / i}^{(\mathcal{M})}+r I_{(T-1)}\right)^{-1}\left(\boldsymbol{y}_{/ i}-\mu\right)+\mu
$$
variance:

589

$$
\psi^{2}=\sigma^{2}+\omega^{2}
$$




$$
\sigma^{2}=\frac{r}{r+1} \psi^{2}, \quad \omega^{2}=\frac{\psi^{2}}{r+1}
$$

593

594 And evaluated the posterior over model parameters using Bayes' rule:

595

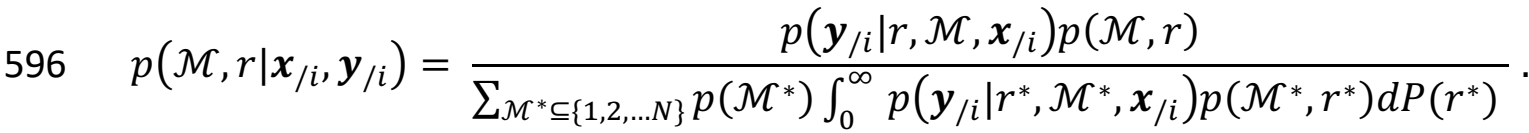

598 We again used Bayes' rule to compute the likelihood term in Eq. 12:

599

600

$$
p\left(\boldsymbol{y}_{/ i} \mid r, \mathcal{M}, \boldsymbol{x}_{/ i}\right)=\frac{p\left(\boldsymbol{y}_{/ i} \mid \mu, \psi^{2}, r, \mathcal{M}, \boldsymbol{x}_{/ i}\right) p\left(\mu, \psi^{2}\right)}{p\left(\mu, \psi^{2} \mid r, \mu, \boldsymbol{x}_{/ i}, \psi^{2}, \mathcal{M}\right)}
$$

601 The likelihood term is computed as in Eq. 4. We again used Bayes' rule to compute the 602 posterior over $\mu$ and $\psi^{2}$ :

603

604

$$
p\left(\mu, \psi^{2} \mid r, \mu, \boldsymbol{x}_{/ i}, \psi^{2}, \mathcal{M}\right) \propto p\left(\boldsymbol{y}_{/ i} \mid \mu, \psi^{2}, r, \mathcal{M}, \boldsymbol{x}_{/ i}\right) p\left(\mu, \psi^{2}\right)
$$

605

606 We then placed a conjugate normal-inverse gamma prior over $\mu$ and $\psi^{2}$ :

607

608

$$
\left(\mu, \psi^{2}\right) \sim \mathrm{N}_{-} \Gamma^{-1}\left(\mu_{0}, \lambda_{0}, \alpha_{0}, \beta_{0}\right)
$$

609

610 where, 
612

$$
\mu_{0}=0 ; \quad \lambda_{0}=1 ; \quad \alpha_{0}=10 ; \quad \beta_{0}=\alpha_{0}+1
$$

613

614 Thus,

615

616

$$
\left(\mu, \psi^{2} \mid r, \mu, \psi^{2}, \mathcal{M}, x_{/ i}\right) \sim \mathrm{N}_{-} \Gamma^{-1}\left(\mu_{\text {post }}^{(i)}, \lambda_{\text {post }}^{(i)}, \alpha_{\text {post }}^{(i)}, \beta_{\text {post }}^{(i)}\right),
$$

617

618 where,

619

620

$$
\mu_{\text {post }}^{(i)}=\frac{b^{(i)}}{a^{(i)}}
$$

621

622

$$
\lambda_{\text {post }}^{(i)}=a^{(i)}
$$

623

624

$$
\alpha_{\text {post }}^{(i)}=\alpha_{0}+\frac{T-1}{2}
$$

625

626

$$
\beta_{\text {post }}^{(i)}=\frac{1}{2}\left(c^{(i)}-\frac{b^{(i)}}{a^{(i)}}\right)
$$

627

628

$$
a^{(i)}=(r+1) \mathbf{1}^{\mathrm{T}}\left(\mathrm{K}_{/ i, / i}^{(\mathcal{M})}+r I\right)^{-1} \mathbf{1}+\lambda_{0}
$$

629

630

$$
b^{(i)}=(r+1) \mathbf{1}^{\mathrm{T}}\left(\mathrm{K}_{/ i, / i}^{(\mathcal{M})}+r I\right)^{-1} \boldsymbol{y}_{/ i},
$$




$$
c^{(i)}=(r+1) \mathbf{1}^{\mathrm{T}}\left(\mathrm{K}_{/ i, / i}^{(\mathcal{M})}+r I\right)^{-1} \boldsymbol{y}_{/ i}+2 \beta_{0}
$$

633

634 where 1 is a vector of ones. With this, we can compute all of the terms in Eq. 13.

635 The integral over Eq. 12 is over one dimension and thus tractable to compute. We chose 636 a discrete distribution for the prior $P(r)$ to increase computation speed:

637

638

$$
P(r)=\text { Uniform }(\{3,4,5.67,9\}),
$$

639

640

such that the GP model could account for $25 \%, 20 \%, 15 \%$ or $10 \%$ of the total variance.

641 We imposed a truncated binomial prior over the number of included features such that

$642|\mathcal{M}| \geq 1$, that favored models with fewer features (i.e., sparse models):

643

644

$$
p(\mathcal{M})=\frac{1}{1-(1-p)^{N}}\left(\begin{array}{c}
N \\
|\mathcal{M}|
\end{array}\right) p^{|\mathcal{M}|}(1-p)^{N-|\mathcal{M}|}
$$

645

646 We set $p=0.1$ so that approximately $2 / 3$ of the prior probability mass rests on single-feature

647 models. We could integrate over a sparse prior in our model, rather than a shrinkage prior such 648 as the Lasso, because we considered only a small $(\mathrm{N}=8)$ set of features (Park and Casella, 2012).

649 Using this normal inverse-gamma description of the posterior, we can compute the prediction 650 of $y_{i}$, given $\mathcal{M}$ and $r$ :

651 


$$
\mathbb{E}\left[y_{i} \mid \mathcal{M}, r, \boldsymbol{x}_{/ i}, \boldsymbol{y}_{/ i}, \boldsymbol{x}_{i}\right]=\mathbb{E}\left[\mathbb{E}\left[y_{i} \mid \mu, \mathcal{M}, r, \boldsymbol{x}_{/ i}, \boldsymbol{y}_{/ i}, \boldsymbol{x}_{i}\right] \mid \mathcal{M}, r, \boldsymbol{x}_{/ i}, \boldsymbol{y}_{/ i}, \boldsymbol{x}_{i}\right]
$$

$$
=\mathrm{K}_{/ i, i}^{(\mathcal{M})^{\mathrm{T}}}\left(\mathrm{K}_{/ i, / i}^{(\mathcal{M})}+r I_{(T-1)}\right)^{-1}\left(\boldsymbol{y}_{/ i}-\mu_{\text {post }}^{(i)}\right)+\mu_{\text {post }}^{(i)}
$$

655

We then insert Eq. 27 and Eq. 12 into Eq. 8 to obtain the prediction of $y_{i}$.

657

658

\section{Construction of the latency distribution}

659 We defined the latency distribution as the set of all latencies between spike bins and song

660 feature windows in which there was a predictive relationship $\left(r^{2}>0\right)$ within the GP model.

\section{Characterizing tuning curves of cell responses}

663 The GP model is flexible in that it will fit any relationship between the independent and

664 dependent variables and is computationally efficient. However, from the output of the model

665 we have no easily interpretable means of characterizing the shape of the fit. In order to

666 characterize the form of the spike-count to song relationships across the large number of fits

667 we assessed, we needed an automated way to categorize the shapes of the tuning curves.

669 a quadratic transformation of the song features (II Memmming Park, 2013). A GLM consists of a

670 stimulus filter, an invertible non-linearity (the link function) and a stochastic exponential non-

671 linearity, such as a Poisson process:

672

$$
y \mid \boldsymbol{x} \sim \operatorname{Poiss}(f(Q(\boldsymbol{x})))
$$

673 


$$
Q(x)=a x^{2}+b x+c
$$

675

676 where $y$ is the spike count, $f$ is the inverse link function, $x$ is the stimulus and $Q$ is a quadratic

677 function of $x$ with coefficients $a, b, c$. We considered song features individually in this tuning

678 curve analysis, so $\operatorname{dim}(\boldsymbol{x})=1$, and the quadratic coefficients became scalars $(a, b, c)$. We took

679 the link function to be an exponential and the noise process to be Poisson. Thus, we can fit the

680 quadratic coefficients by maximizing the log-likelihood:

681

682

$$
\log P(Y \mid X, a, b, c)=\sum_{i=1}^{N}\left[-\exp \left(Q\left(x_{i}\right)\right)+a * y_{i} x_{i}^{2}+b * y_{i} x_{i}+c-\log \left(\mathrm{y}_{\mathrm{i}} !\right)\right]
$$

683 We maximized the log-likelihood numerically using conjugate gradient methods. The sign of the

684 quadratic coefficient, a, of this model determines whether the data is better fit by an upwards-

685 facing, quadratic basis in which the data is double-peaked, or a downwards-facing quadratic

686 basis in which the data is single-peaked. We compared this model to a nested model fit where

687 the quadratic term is set to zero (I-GLM).

688

We compared the performance of the two models using the Akaike information

689 criterion (AIC) (Akaike, 1974). The AIC metric is defined as:

690

691

$$
A I C=2 k-2 \ln \hat{\mathcal{L}}
$$

692

693

$$
\hat{\mathcal{L}}=\operatorname{argmax}_{a, b, c} \log P(Y \mid X, a, b, c),
$$

694 
695 where $\hat{\mathcal{L}}$ is the maximum of the log-likelihood function for a given model and $k$ is the number of 696 estimated parameters. This metric balances goodness of fit with model complexity. A lower AIC 697 metric indicates better performance. Therefore, the difference in the AIC metrics of two models

698 indicates the relative success of one model over another, taking into account differences in 699 model complexity (Akaike, 1974; Raftery, 1995). We can then ask, when the quadratic model

700 (q-GLM) is a better fit to the data than the linear model (I-GLM), does the tuning curve 701 relationship of spike counts to song features show a positive or negative curvature? We 702 predicted that a PPE-like signal should have a negative curvature.

703 We compared the q-GLM and I-GLM models on all GP model fits with $r^{2}>0$ for all 704 individual song features which had themselves predictive fits within the multi-dimensional 705 model. We calculated the fraction of fits with the quadratic coefficient, a $<0$, as a function of 706 the AIC comparison between the two models:

707

708

$$
\Delta A I C \equiv 2 k_{\text {linear }}-2 \ln \hat{\mathcal{L}}_{\text {linear }}-2 k_{\text {quad }}+2 \ln \hat{\mathcal{L}}_{\text {quad }}
$$

\section{Evaluating the Gaussian Process model performance}

713 To evaluate GP model performance we use a leave-one-out cross validation method to estimate 714 the mean-squared prediction error for new observations as in (Vehtari, 2017): 


$$
M S E_{\text {loo }}^{(G P)}=\frac{1}{T} \sum_{i=1}^{T}\left(y_{i}-\hat{y}_{i}\right)^{2},
$$

$$
\hat{y}_{i}=\mathbb{E}\left[y_{i} \mid \boldsymbol{x}_{/ i}, \boldsymbol{y}_{/ i}, \boldsymbol{x}_{i}\right],
$$

720 where $\hat{y}_{i}$ is the model prediction spike count for rendition ' $i$ ', and $\boldsymbol{x}_{/ i}$ and $\boldsymbol{y}_{/ i}$ are the song

721 features and spike counts of all renditions excluding the $\mathrm{i}^{\text {th }}$ rendition. We then compared the GP

722 model to a model with constant mean firing rate equal to the mean spike count over all

723 renditions excluding the $\mathrm{i}^{\text {th }}$ rendition:

724

725

$$
y_{(i)} \sim \mathcal{N}\left(\alpha, \tau^{2}\right) .
$$

726 The predictive mean-squared error of this model is:

727

728

$$
M S E_{\text {loo }}^{(\text {null })}=\frac{1}{T} \sum_{i=1}^{T}\left(y_{i}-\overline{y_{/ l}}\right)^{2}
$$

729 where,

730

731

$$
\overline{y_{/ \iota}}=\frac{1}{T-1} \sum_{j \neq i} y_{j} .
$$

732

733 The cross-validated $r^{2}$ value is: 


$$
r^{2}=1-\frac{M S E_{l o o}^{(G P)}}{M S E_{l o o}^{(\text {null })}}
$$

737 An $r^{2}>0$ indicates that the model predicts new observations better than simply using the

738 mean. The maximum theoretical value the $r^{2}$ can take is one-this indicates perfect model 739 prediction and, in practice, is never achieved. We use the $r^{2}$ value as our measure of model 740 performance.

\section{Bootstrapping to assess population-level significance}

743 Assessing the significance of the model predictions must be done on a population level for this

744 type of analysis. We generated model fits to hundreds of song segment-spike count pairs for

745 each syllable. Simply by chance, a portion of these fits would generate a predictive $r^{2}>0$

746 value.

$747 \quad$ Furthermore, spike-song pairs are correlated, not only because of overlapping spike

748 counts and song segment windows but also because of possible underlying correlations in the

749 song and spike fluctuations across the song. To address this, we randomized the relationship

750 between entire spike trains and song renditions and then re-performed our model fits on the

751 randomized, spike count-song segment pairs across all time steps. By leaving the temporal

752 structure of the song and spiking activity intact and only randomizing the relationship between

753 them, we built a randomized population of fits for each cell-syllable pair, which retained the

754 unknown, underlying temporal structure possibly present in the spike trains and song (Tusher 
755

756

757

758

759

760

761

762

763

764

765

766

767

768

769

770

771

772

773

774

775

776

et al., 2001). We repeated this procedure 100 times for the VTA error cell population and 100

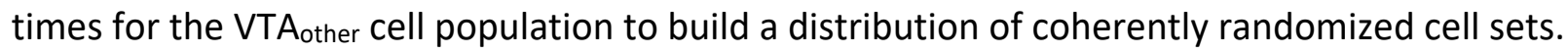
From this distribution of randomized cell sets, we computed single-tailed p-values assessments of the $r^{2}$ values of the individual spike count-song segment model fits as well as on population measures of significance in the VTA $A_{\text {error }}$ and VTA other $_{\text {cell }}$ populations independently. We assessed four population measures:

1. The frequency of the predictive signal across the whole cell population. An $r^{2}>$ 0 indicates the model predicts the data better than an estimate based solely on the mean spike count across renditions, and we call this a 'predictive signal'. We therefore assessed the significance of the total number of $r^{2}>0$ song segment-spike count fits within the PPE latency window for both the VTA error $(p<0.01)$ and VTA other $(p<0.01)$ syllable-cell pairs respectively with a single-tailed $p$-value test.

2. The spread of the predictive signal across the cell population. We asked whether a small number of cells were accounting for the majority of the positive $r^{2}$ values or if the signal appeared across multiple cells and syllables in the population. To answer this, we first labeled each cell-syllable pair as 'significant' if the number of positive $r^{2}$ values within the PPE latency window $(0-150 \mathrm{~ms})$ had a single-tailed $p<0.05$. We then calculated the single-tailed $p$-value for the number of significant cell-syllable pairs across the entire cell population (VTA error population: one-sided z-test: $p<0.01$; VTA other population: onesided z-test: $p<0.01)$.

3. The significance of the magnitude of the peak in signal occurrence within the PPE latency window across the cell population. We computed latency distributions as the latencies 
of the full set of spike-count song feature pairs that resulted in GP model fits with $r^{2}>0$. We compared the variance of the latency distributions of the randomized populations to the variance of the peak we found in the actual data. We computed the single-tailed pvalue for the maximum fluctuation of a latency distribution at any point in the latency domain. In this way we tested the significance not only of finding a peak in the data at the PPE window but of finding a peak of that size anywhere in the latency distribution. For example, VTA error population latency distribution had a peak within the expected PPE latency region (Figure $3 C$ ). This peak was 3.84 standard deviations from the mean. The variance in relation to the maximum variance in randomized latency distributions was significant (one-sided z-test: $p<0.01$ ). The time-bin of this latency distribution was


from the mean (Figure 3D). This variance was not significant (one-sided $z$-test: $p=0.21$ ). The time-bin of this latency distribution was $25 \mathrm{~ms}$.

4. The significance of the shapes of tuning curves we find via our GLM parameterization technique. We computed the single-tailed p-value of the fraction of single-peaked tuning curves in the real population relative to the randomized populations (Figure 4E legend and main text). Note that the goal of this significance strategy allows us to assess the VTAerror cell 795 activity as a population, not the significance of particular song segment-spike count pairs. More 796 data are needed for this level of specificity. 

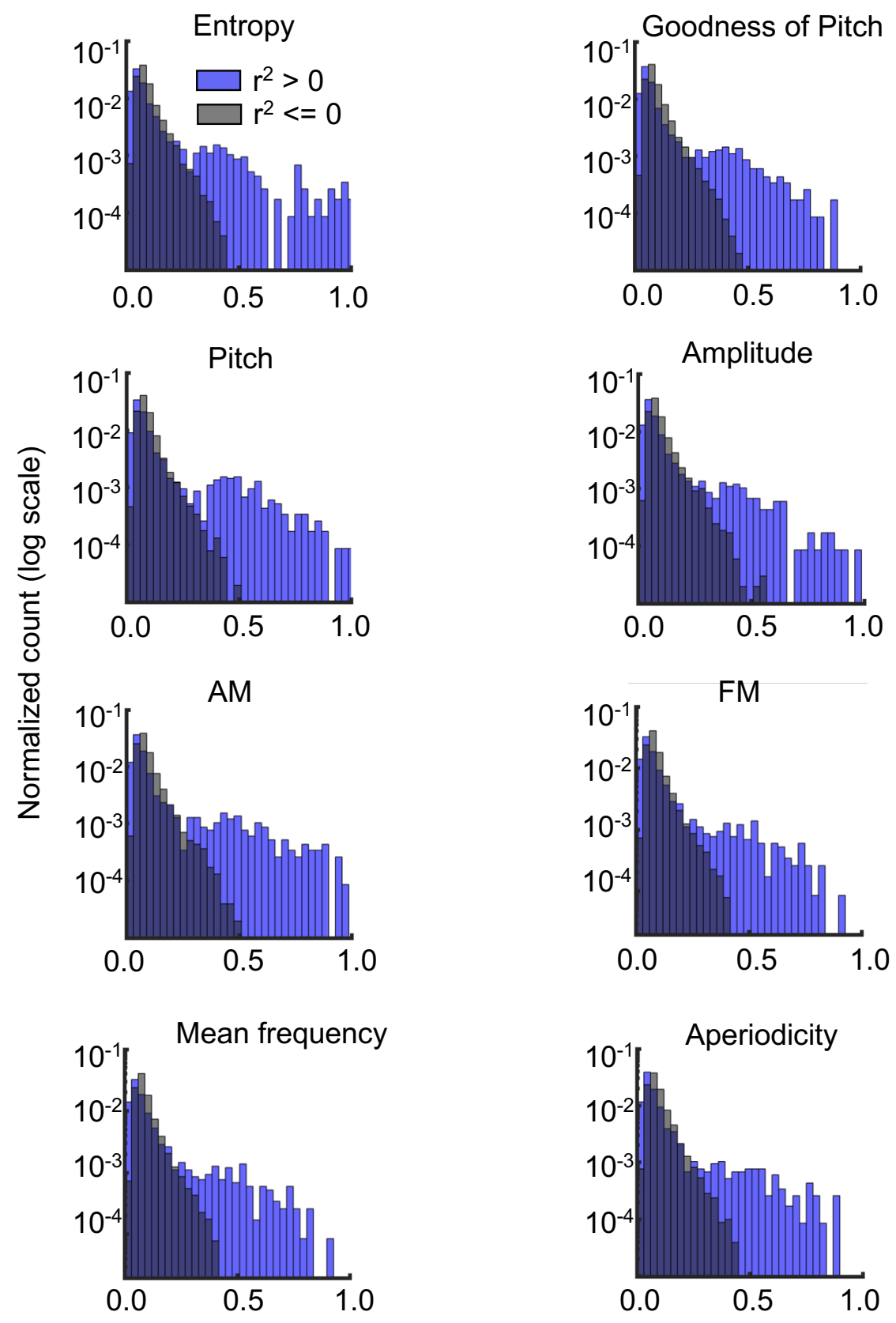

Feature weight in GP model

Figure S1 


\section{Figure S1. Related to Figure 2. Spiking Responses to Song Fluctuations are Captured by a}

\section{Subset of Available Features, which Varies Across Context}

801 Distributions of individual song feature weights in the full GP model for $r^{2}>0$ and $r^{2}<=0$

802 populations. In fits with $r^{2}>0$ (predictive), the distribution was bimodal with an additional peak

803 at around 0.5 , implying that in predictive fits fewer numbers of features captured most of the

804 information. In overfit models $\left(r^{2}<=0\right)$ all features contributed more equally to the poor

805 estimate. Also note that the distributions across features were quite similar; no one feature

806 captured significantly more of the song variations. 

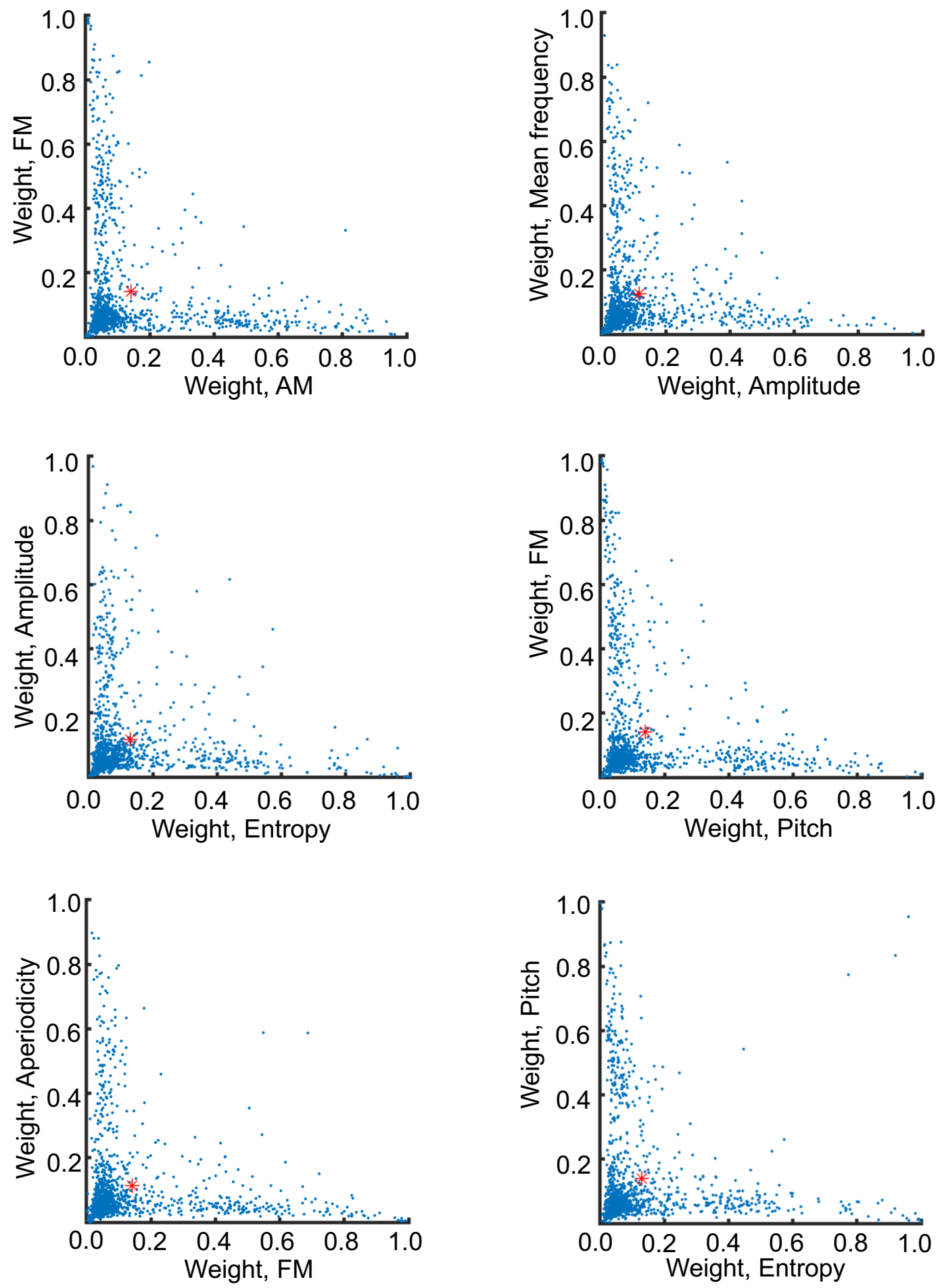

Figure S2 


\section{Figure S2. Related to Figure 2. Feature Contributions to Full GP Model Show Pairwise}

809 Correlations at Low, But Not High Weights

810 Scatterplots of relationships of feature contributions to the full GP model for individual fits with

$811 r^{2}>0$ within the PPE window. All plots: each point represents one model fit with an $r^{2}>0$. Axes

812 are the total feature weight in full the GP model of indicated feature; red dot is mean value.

813 Pairs of features were selected to be representative of the full model set. All pairs showed

814 comparable correlations at low weights. 

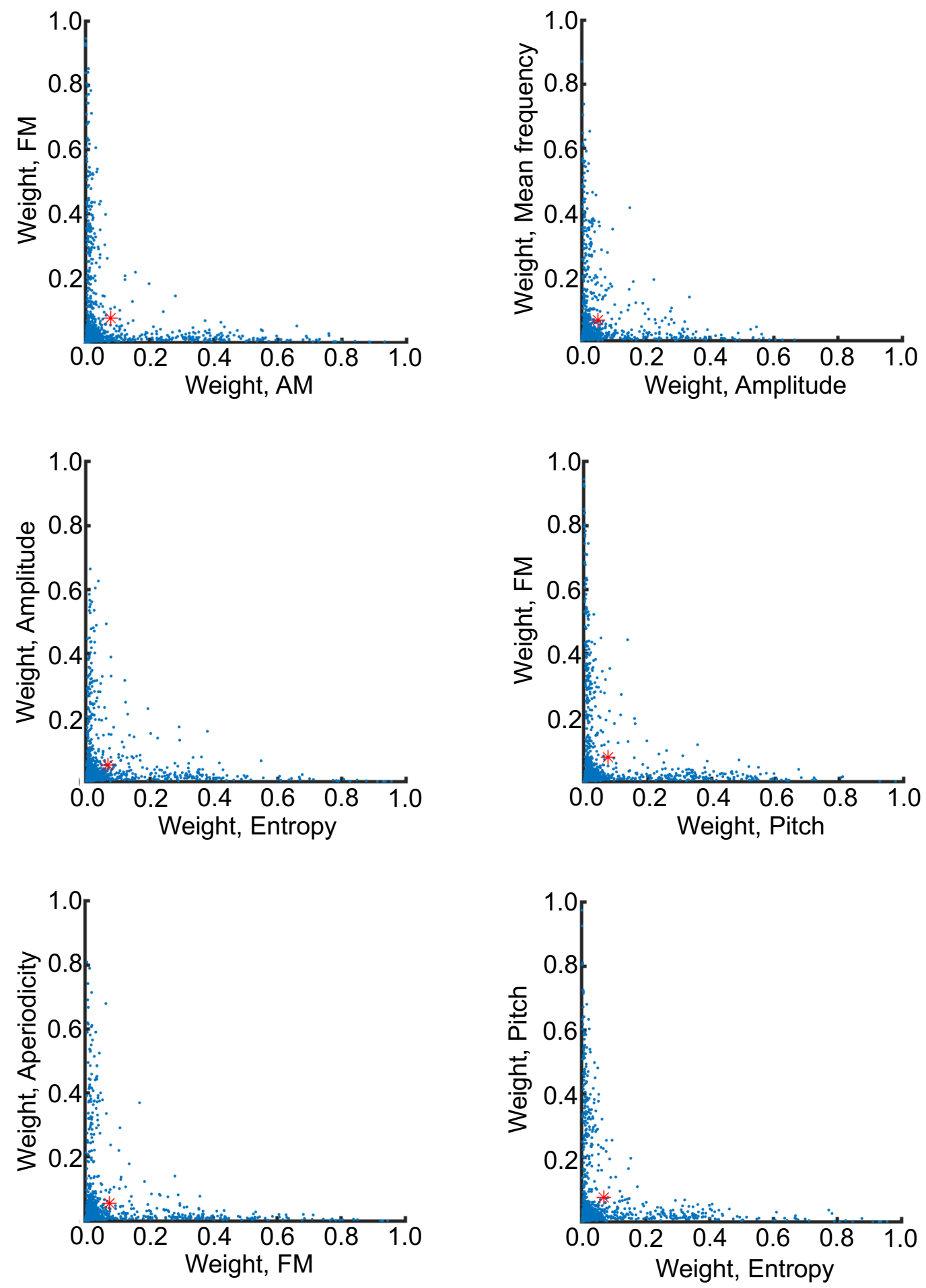

Figure S3 


\section{Figure S3. Related to Figure 2. $1^{\text {st }}$ Order Feature Contributions are Not Correlated}

817 Scatterplots of Relationships of only the $1^{\text {st }}$ order feature contributions to the full GP model for 818 individual fits with $r^{2}>0$ within the PPE window. All plots: each point represents one model fit 819 with an $r^{2}>0$. Axes are the $1^{\text {st }}$ order feature weights in full GP model; red dot is mean value.

820 Pairs of features were selected to be representative of the full model set. These data showed 821 that correlations between features are mainly higher order coupling effects. 
A

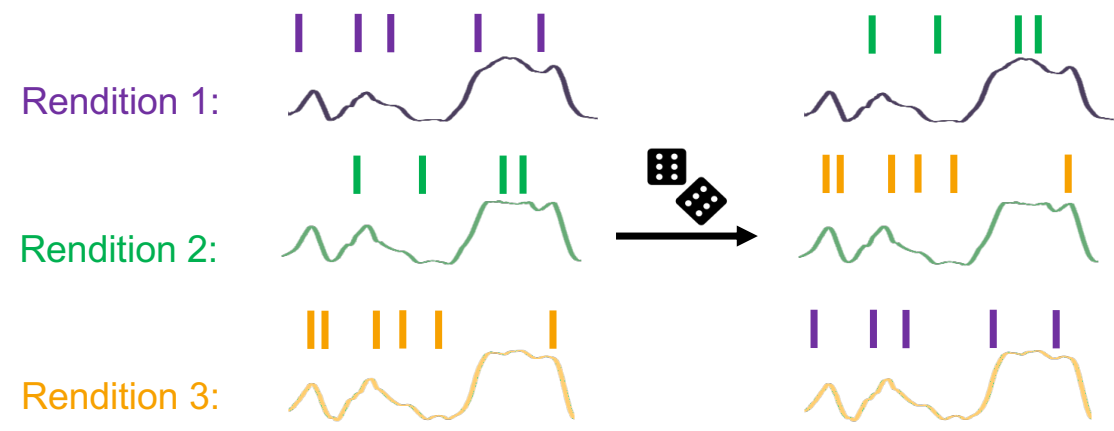

B

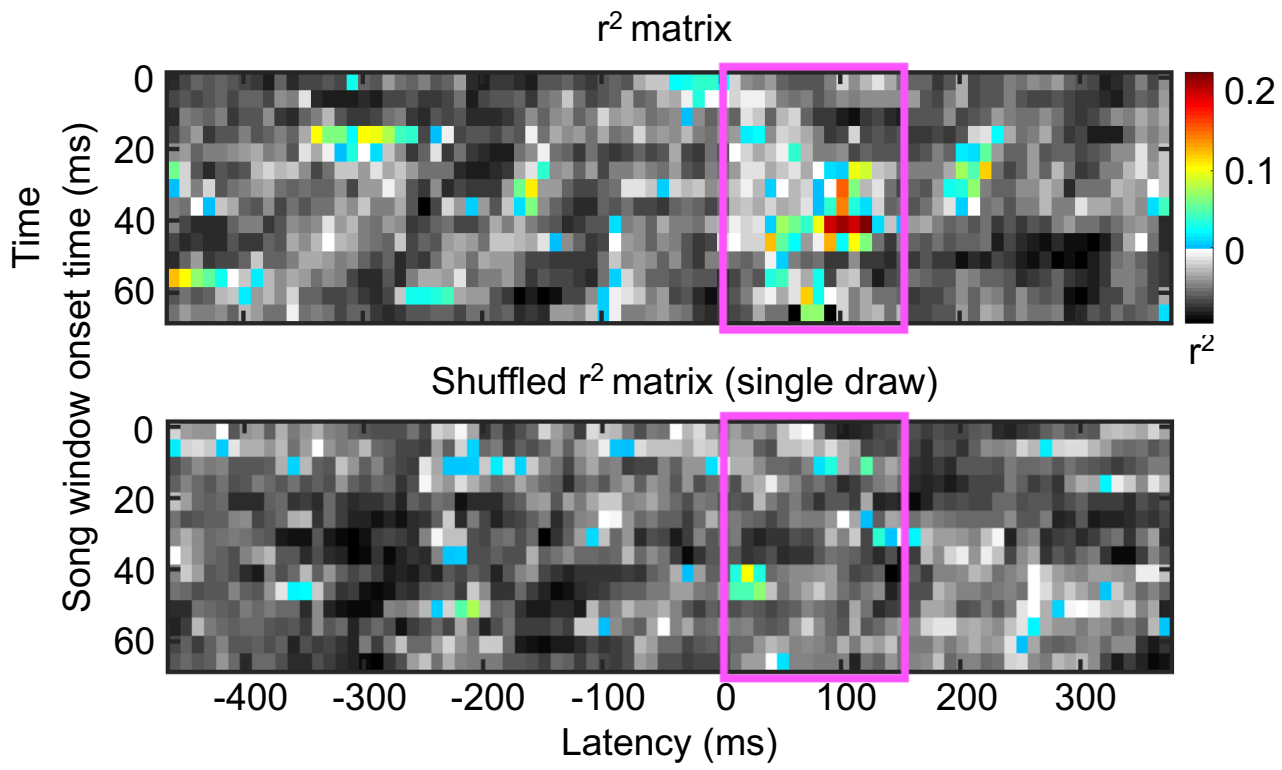

C

Fraction $r^{2}>0: 0.090$

Fraction $r^{2}$ shuffled $>0: 0.091$



Fraction $r^{2}>0: 0.200$

Fraction $r^{2}$ shuffled $>0: 0.095$

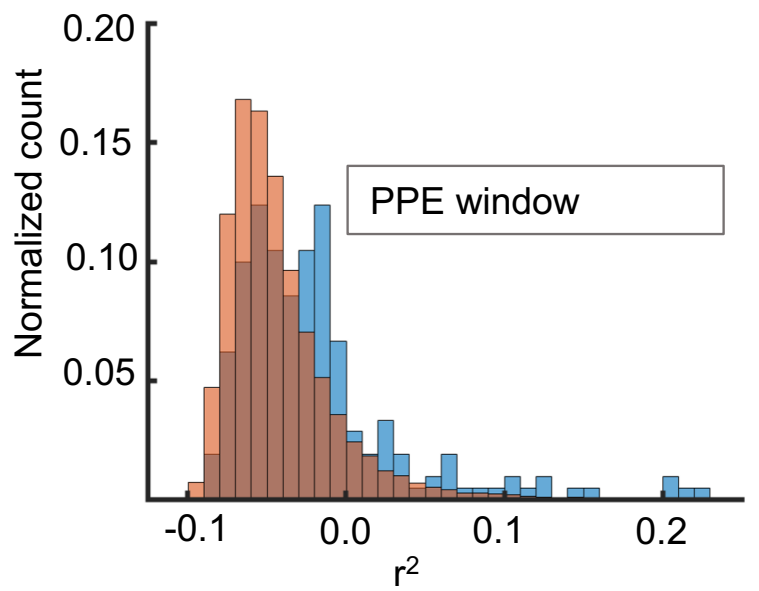

Figure S4 


\section{Figure S4. Related to Figure 3. Coherent Shuffling of Entire Spike Trains Retains Underlying}

\section{Correlation Structure and Permits a Population-level Significance Assessment}

825 (A) Schematic of spike train shuffling. Spike trains were randomized relative to the associated

826 song. The randomized song-spike relationship was re-fit retaining possible underlying

827 correlations in the spike train and song fluctuations.

828 (B) Panel replicated from Figure $3 A$.

829 (C) $r^{2}$ distributions for randomized and actual data. Left: $r^{2}$ distributions for the shuffled and real

830 data compared across all latencies. The real and shuffled distributions appeared quite similar.

831 The number of $r^{2}>0$ in the real data was not significantly different from what would be

832 expected by chance. Right: the distribution of $r^{2}$ values that fall within the PPE latency window

833 compared to the randomized distribution from within this same latency range. This distribution

834 was shifted away from the randomized distribution, with more, larger $r^{2}>0$. This population

835 had a significantly greater number of $r^{2}>0$ than expected by chance (one tailed z-test, $p$-value $=$

836 0.02).

837

838

839

840

841

842

843

844 
846

847

848

849

850

851

852

853

854

855

856

857

858

859

860

861

862

863

864

865

866

867

868

869

870

871

872

873

874

875

876

877

878

879

880

881

882

883

884

885

886

Akaike, H. (1974). A new look at the statistical model identification. IEEE Transactions on Automatic Control 19, $716-723$.

Ali, F., Otchy, T.M., Pehlevan, C., Fantana, A.L., Burak, Y., and Olveczky, B.P. (2013). The basal ganglia is necessary for learning spectral, but not temporal, features of birdsong. Neuron 80 , 494-506. 10.1016/j.neuron.2013.07.049.

Andalman, A.S., and Fee, M.S. (2009). A basal ganglia-forebrain circuit in the songbird biases motor output to avoid vocal errors. Proc Natl Acad Sci U S A 106, 12518-12523. 10.1073/pnas.0903214106.

Barter, J.W., Li, S., Lu, D., Bartholomew, R.A., Rossi, M.A., Shoemaker, C.T., Salas-Meza, D., Gaidis, E., and Yin, H.H. (2015). Beyond reward prediction errors: the role of dopamine in movement kinematics. Front Integr Neurosci 9, 39. 10.3389/fnint.2015.00039.

Brainard, M.S., and Doupe, A.J. (2000). Interruption of a basal ganglia-forebrain circuit prevents plasticity of learned vocalizations. Nature 404, 762-766. 10.1038/35008083.

Chen, R., Gadagkar, V., Roeser, A.C., Puzerey, P.A., and Goldberg, J.H. (2021). Movement signaling in ventral pallidum and dopaminergic midbrain is gated by behavioral state in singing birds. J Neurophysiol. 10.1152/jn.00110.2021.

Cohen, J.Y., Haesler, S., Vong, L., Lowell, B.B., and Uchida, N. (2012). Neuron-type-specific signals for reward and punishment in the ventral tegmental area. Nature 482, 85-88.

10.1038/nature10754.

da Silva, J.A., Tecuapetla, F., Paixão, V., and Costa, R.M. (2018). Dopamine neuron activity before action initiation gates and invigorates future movements. Nature. 10.1038/nature25457. Deregnaucourt, S., Mitra, P.P., Feher, O., Maul, K.K., Lints, T.J., and Tchernichovski, O. (2004). Song development: in search of the error-signal. Ann N Y Acad Sci 1016, 364-376. 10.1196/annals.1298.036.

Dobi, A., Margolis, E.B., Wang, H.L., Harvey, B.K., and Morales, M. (2010). Glutamatergic and nonglutamatergic neurons of the ventral tegmental area establish local synaptic contacts with dopaminergic and nondopaminergic neurons. J Neurosci 30, 218-229.

10.1523/JNEUROSCI.3884-09.2010.

Engelhard, B., Finkelstein, J., Cox, J., Fleming, W., Jang, H.J., Ornelas, S., Koay, S.A., Thiberge, S.Y., Daw, N.D., Tank, D.W., and Witten, I.B. (2019). Specialized coding of sensory, motor and cognitive variables in VTA dopamine neurons. Nature 570, 509-513. 10.1038/s41586-019-12619.

Gadagkar, V., Puzerey, P.A., Chen, R., Baird-Daniel, E., Farhang, A.R., and Goldberg, J.H. (2016). Dopamine neurons encode performance error in singing birds. Science 354, 1278-1282.

$10.1126 /$ science.aah6837.

Goffinet, J., Brudner, S., Mooney, R., and Pearson, J. (2021). Low-dimensional learned feature spaces quantify individual and group differences in vocal repertoires. bioRxiv, 811661 . 10.1101/811661.

Hamaguchi, K., Tschida, K.A., Yoon, I., Donald, B.R., and Mooney, R. (2014). Auditory synapses to song premotor neurons are gated off during vocalization in zebra finches. Elife 3 , e01833. 10.7554/eLife.01833. 
Hamid, A.A., Pettibone, J.R., Mabrouk, O.S., Hetrick, V.L., Schmidt, R., Vander Weele, C.M., Kennedy, R.T., Aragona, B.J., and Berke, J.D. (2016). Mesolimbic dopamine signals the value of work. Nat Neurosci 19, 117-126. 10.1038/nn.4173. Hamilos, A.E., Spedicato, G., Hong, Y., Sun, F., Li, Y., and Assad, J.A. (2020). Dynamic dopaminergic activity controls the timing of self-timed movement. bioRxiv, 2020.2005.2013.094904. 10.1101/2020.05.13.094904.

Hisey, E., Kearney, M.G., and Mooney, R. (2018). A common neural circuit mechanism for internally guided and externally reinforced forms of motor learning. Nat Neurosci 21, 589-597. 10.1038/s41593-018-0092-6. Hoeting, J.A., Madigan, D., Raftery, A. E., \& Volinsky, C. T. (1998). Bayesian model averaging Proceedings of the AAAI Workshop on Integrating Multiple Learned Models 335, 77-83. Hoffmann, L.A., Saravanan, V., Wood, A.N., He, L., and Sober, S.J. (2016). Dopaminergic Contributions to Vocal Learning. J Neurosci 36, 2176-2189. 10.1523/JNEUROSCI.3883-15.2016. Howe, M.W., Tierney, P.L., Sandberg, S.G., Phillips, P.E., and Graybiel, A.M. (2013). Prolonged dopamine signalling in striatum signals proximity and value of distant rewards. Nature $500,575-$ 579. 10.1038/nature12475.

Il Memmming Park, E.A., Nicholas Priebe, \& Jonathon Pillow (2013). Spectral methods for neural characterization using generalized quadratic models. Advances in Neural Information Processing Systems 26, 2454-2462. Ju Tian, R.H., Jeremiah Y. Cohen, Fumitaka Osakada, Dmitry Kobak, Christian K. Machens, Edward M. Callaway, Naoshige Uchida, and Mitsuko Watabe-Uchida (2016). Distributed and Mixed Information in Monosynaptic Inputs to Dopamine Neurons. Neuron 91, 1374-1389. Kao, M.H., Doupe, A.J., and Brainard, M.S. (2005). Contributions of an avian basal gangliaforebrain circuit to real-time modulation of song. Nature 433, 638-643. 10.1038/nature03127. Kao, M.H., Wright, B.D., and Doupe, A.J. (2008). Neurons in a forebrain nucleus required for vocal plasticity rapidly switch between precise firing and variable bursting depending on social context. The Journal of neuroscience : the official journal of the Society for Neuroscience 28, 13232-13247. 10.1523/JNEUROSCI.2250-08.2008.

Kollmorgen, S., Hahnloser, R.H.R., and Mante, V. (2020). Nearest neighbours reveal fast and slow components of motor learning. Nature 577, 526-530. 10.1038/s41586-019-1892-x.

Leblois, A., Wendel, B.J., and Perkel, D.J. (2010). Striatal dopamine modulates basal ganglia output and regulates social context-dependent behavioral variability through D1 receptors. J Neurosci 30, 5730-5743. 10.1523/JNEUROSCI.5974-09.2010. Lipkind, D., and Tchernichovski, O. (2011). Quantification of developmental birdsong learning from the subsyllabic scale to cultural evolution. Proc Natl Acad Sci U S A 108 Suppl 3, 1557215579. 10.1073/pnas.1012941108.

Mandelblat-Cerf, Y., Las, L., Denisenko, N., and Fee, M.S. (2014). A role for descending auditory cortical projections in songbird vocal learning. Elife 3. 10.7554/eLife.02152.

Park, T., and Casella, G. (2012). The Bayesian Lasso. Journal of the American Statistical Association 103, 681-686. 10.1198/016214508000000337. Raftery, R.E.K.A.E. (1995). Bayes Factors. Journal of American Statsitical Association 90, 773795. 
929 Ravbar, P., Lipkind, D., Parra, L.C., and Tchernichovski, O. (2012). Vocal exploration is locally 930 regulated during song learning. The Journal of neuroscience : the official journal of the Society 931 for Neuroscience 32, 3422-3432. 10.1523/JNEUROSCI.3740-11.2012.

932 Schultz, W., Dayan, P., and Montague, P.R. (1997). A neural substrate of prediction and reward. 933 Science 275, 1593-1599. 10.1126/science.275.5306.1593.

934 Sober, S.J., and Brainard, M.S. (2009). Adult birdsong is actively maintained by error correction. 935 Nat Neurosci 12, 927-931. 10.1038/nn.2336.

936 Sutton, R.S., and Barto, A.G. (1998). Reinforcement learning: an introduction (MIT Press).

937 Tchernichovski, O., Nottebohm, F., Ho, C.E., Pesaran, B., and Mitra, P.P. (2000). A procedure for 938 an automated measurement of song similarity. Anim Behav 59, 1167-1176.

939 10.1006/anbe.1999.1416.

940 Tumer, E.C., and Brainard, M.S. (2007). Performance variability enables adaptive plasticity of

941 'crystallized' adult birdsong. Nature 450, 1240-1244. 10.1038/nature06390.

942 Tusher, V.G., Tibshirani, R., and Chu, G. (2001). Significance analysis of microarrays applied to 943 the ionizing radiation response. Proc Natl Acad Sci U S A 98, 5116-5121.

944 10.1073/pnas.091062498.

945 Vehtari, A., Gelman, A., \& Gabry, J. (2017). Practical bayesian model evaluation using leave-one-

946 out cross-validation and waic. Statistics and Computing 27 (5), 1413-1432.

947 Williams, C.K.a.R., C.E. (2006). Gaussian Processes for Machine Learning (The MIT Press).

948 Wood, J., Simon, N.W., Koerner, F.S., Kass, R.E., and Moghaddam, B. (2017). Networks of VTA

949 Neurons Encode Real-Time Information about Uncertain Numbers of Actions Executed to Earn a 950 Reward. Front Behav Neurosci 11, 140. 10.3389/fnbeh.2017.00140.

951 Woolley, S.C., and Kao, M.H. (2014). Variability in action: Contributions of a songbird cortical952 basal ganglia circuit to vocal motor learning and control. Neuroscience.

953 10.1016/j.neuroscience.2014.10.010.

954 Xiao, L., Chattree, G., Oscos, F.G., Cao, M., Wanat, M.J., and Roberts, T.F. (2018). A Basal

955 Ganglia Circuit Sufficient to Guide Birdsong Learning. Neuron 98, 208-221 e205.

956 10.1016/j.neuron.2018.02.020.

957 